Metaliurgical Engineering Department
Ohio State University
Columbus, Ohio 43210

and

R. W. Balluffi

Department of Materials Science and Engineering

Cornell University

Ithaca, New York 14850

27 September 1972

Cornell University

I thaca, New York

THIS DOCUMENT CONFIRMED AS

UNCLASSIFIED

DIVISION OF CLASSIFICATION

BY JaCK H. Kabn/wer
DATE 11/6/92
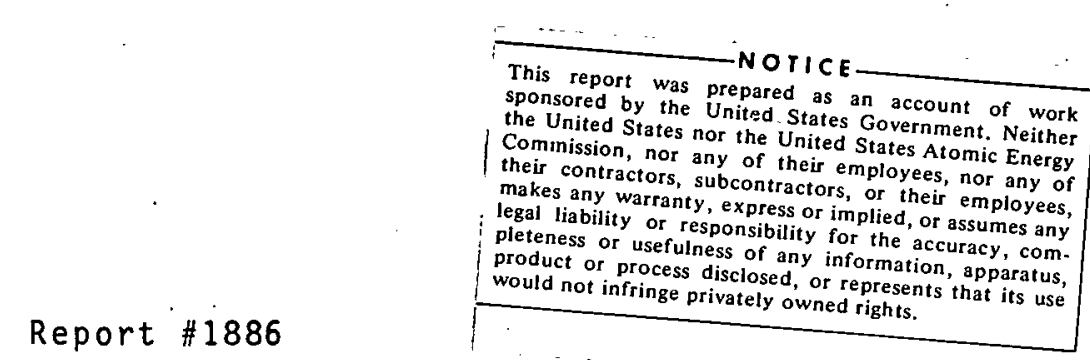

Is sued by

The Materials Science Center

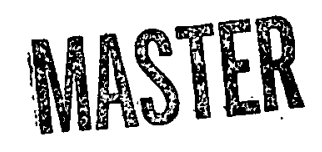

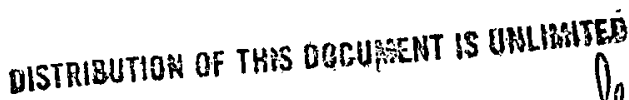




\section{DISCLAIMER}

This report was prepared as an account of work sponsored by an agency of the United States Government. Neither the United States Government nor any agency Thereof, nor any of their employees, makes any warranty, express or implied, or assumes any legal liability or responsibility for the accuracy, completeness, or usefulness of any information, apparatus, product, or process disclosed, or represents that its use would not infringe privately owned rights. Reference herein to any specific commercial product, process, or service by trade name, trademark, manufacturer, or otherwise does not necessarily constitute or imply its endorsement, recommendation, or favoring by the United States Government or any agency thereof. The views and opinions of authors expressed herein do not necessarily state or reflect those of the United States Government or any agency thereof. 


\section{DISCLAIMER}

Portions of this document may be illegible in electronic image products. Images are produced from the best available original document. 


\title{
ON GRAIN BOUNDARY DISLOCATIONS AND LEDGES*
}

\author{
by \\ J. P. Hirth \\ Metallurgical Engineering Department \\ Ohio State University \\ Columbus, Ohio 43210 \\ and \\ R. W. Balluffi \\ Department of Materials Science and Engineering \\ Cornell liniversity \\ I thaca, New York 14850
}

\section{ABSTRACT}

Models for line defects. in grain boundaries are reviewed. The structures of grain boundary dislocations (GBD's) and ledges are examined, and line defects which correspond to either pure GBD's, pure grain boundary ledges or else defects possessing both dislocation and ledge character are distinguished. The concept of impotent grain boundary dislocations (IGBD's) is introduced, and circuits are defined which yield the Burgers vectors of both GBD's and IGBD's. Two distinct types of circuits are found to be useful and are described. Virtual grain boundary dislocations (VGBD's) are also considered. What have been called VGBD's are shown in some cases to be true GBD's. In other cases, defects exist which could logically be termed VGBD's, but these can often alternatively be regarded as the result of the superposition of several GBD's or IGBD's. GBD's formed in grain boundary cutting or impingement processes are found to be identical to secondary GBD's in boundaries of near-coincidence orientation. Generalized secondary GBD's are found to represent an intermediate representation between the Frank model and the surface dislocation model.

* The research was supported in part (J.P.H.) by the National Science Foundation under Grant GK 18770. Additional support (R.W.B.) was received from the U.S. Atomic Energy Commission under Contract AT(11-1) 3158 and the Advanced Research Projects Agency through the Materials Science Center at Cornell University. 


\section{INTRODUCTION}

In describing line defects in grain boundaries, the terms steps, ledges, primary dislocations, secondary dislocations, infinitesimal dislocations, surface dislocations, grain boundary dislocations (GBD's), and virtual grain boundary dislocations (VGBD's) have all been used, sometimes in an ambiguous manner. The purpose of the present paper is to investigate in some detail the topological properties of grain boundary dislocations and ledges. In particular, we are concerned with developing in one place a formal framework for analyzing these defects in a systematic and self-consistent manner. In the course of the work the basic types of line defects which appear to be useful for this purpose are defined, and a systematic nomenclature for them is developed. In addition, we discuss several postulates with regard to GBD's, in particular with respect to processes in which lattice dislocations cut through or impinge upon grain boundaries.

\section{GRAIN BOUNDARY DISLOCATIONS VERSUS LEDGES (STEPS)}

It is important in any consideration of line defects in grain boundaries to distinguish clearly between pure GBD's, pure grain boundary ledges (steps)* and line defects which possess both dislocation and ledge characters. In the early literature ${ }^{(1-3)}$ on GBD's the expression grain boundary ledge was used synonymously with GBD. While ledges are often associated with GBD's, they need not be, so, as noted elsewhere(4), the interchangeable usage should be dropped and Ashby's notation for ledges adopted $(5)$. As has been emphasized recently ${ }^{(6)}$, the topology of GBD's and grain. boundary ledges can be investigated simply by hypothetical cuts and displacements analogous to those used to describe Volterra dislocations in perfect crystals. Imagine first a reference bicrystal completely free of GBD's as in Fig. Ib which is produced by joining together two crystals of differing orientation (Crystals 1 and 2 ) possessing macroscopically flat surfaces ( $A B$ and $C D$ ) as shown in Fig. 1a. A GBD is introduced by making a cut along the original

* We shall regard the terms ledge and step as synonymous. 
joining plane EF down to the point $P$ and displacing the two sides of the cut by the Burgers vector, $\vec{b}$. If the two sides of the cut can now be joined together, without further displacements relative to each other, in order to form a boundary which is indistinguishable from the original boundary, a total GBD will have been introduced into the boundary at $P$. On the other hand, if a new boundary with differing structure is produced the defect will be a partial GBD, and the new boundary area which bounds one side of the dislocation may be regarded as a faulted boundary. In the case where the displacement across the cut has a component normal to the boundary, it is, of course, necessary to add (subtract) material in the usual way. to produce the join (displacement) across the cut as seen in Figs. $1 c$, d. The Burgers vector can be found by comparing appropriate circuits in the reference bicrystal and the defect bicrystal. Precautions in the choice of the circuit are usually necessary, however, as discussed in a later section.

In the example shown in Fig. 1d the line defect has both ledge and dislocation characters. The offset in the boundary at the defect may be regarded as a ledge, since the migration of this line defect in the boundary plane either adds or subtracts an atomic plane from Crystal 1 causing the boundary to be displaced with respect to Crystal 1 in a direction normal to itself. However, the line defect also has GBD character, since a Burgers circuit around it exhibits closure failure, and we may therefore regard the line defect as a GBD which also possesses ledge character. We note that the displacement (Burgers vector) in Fig. 1d may, or may not, have a component parallel to the boundary.*. In either case climb is necessary for motion of the ledge in the boundary plane, and we therefore call it a climb ledge or c-ledge. Another line defect is illustrated in Figs. le, f. This defect clearly has ledge character. Here the heights of the initial ledges on crystals 1 and 2 are identical in Fig. 1e, and a displacement parallel to the

* An example of a case where the GBD-ledge has a small component parallel to the boundary is given by the dislocation with Burgers vector $\vec{b}_{8}$ in $\mathrm{Fig} .8$ of $\operatorname{Ref.} 40$. 
boundary either may, or may not, be necessary to make the proper join (Fig. 1f).

When a parallel displacement is necessary we again have a GBD which also possesses ledge character. However, in this case the line defect can move. in the boundary plane by the transfer of atoms from one crystal to the other locally at the ledge together with glide of the GBD. Since the motion of the ledge can be accomplished without climb, we call it a glide ledge or G-ledge. Examples of GBD's with G-ledge character include twinning disloca-. tions in coherent twin boundaries (7) (see section 4) and GBD's which act as ledges in precipitate growth such as that of the $\theta^{\prime}$ phase in aluminum-copper alloys (8).

When no parallel displacement in the boundary plane is necessary in the latter case we obtain a pure ledge. Such a defect completely lacks dislocation character in the conventional sense*, and it can migrate by the transfer of atoms from one crystal to the other locally at the ledge. Under these conditions the boundary is displaced with respect to both crystals, and no relative motion of one crystal with respect to the other occurs. As Ashby showed ${ }^{(5)}$, ledges can exist at equilibrium interphase interfaces and can move by diffusion processes, in both cases without associated dislocations, Fig. 2. An analogous situation for a one component system is the growth of a vicinal surface into the vapor phase by the motion of surface ledges $(9)$.

Finally, a pure GBD, without ledge character is readily produced. For example, if no ledge is present on either crystal of Fig. 1c, and if the displacement across the cut is paraliel to the boundary, the result is a pure GBD.

There are several interesting relationships between grain boundary ledges and dislocations. For example, there is the possibility, because of local atomic relaxations at ledges, that ledges can act as sources for lat-

* As seen later, however, such a ledge may possess "impotent" dislocation character. 
tice dislocations which may, or may not, incorporate a GBD formerly associated with a ledge $(10,11)$. Even in this case, however, GBD's are not required. An example is shown in Fig. 3 which illustrates a domain boundary between clustered regions in an $A B$ system. In the usual quasichemical description, in the extreme case that the depicted vertical $A B$ bond has an energy greater than that of a dislocation pair, the GBD-free ledge can relax as shown, emitting lattice dislocations. An analog of this case involving spontaneous punching-in of a surface ledge at a crystal-vapor interface to form a dislocation is suggested by the work of Herring(12), and others $(13,14)$. In the context of the discussion in the previous example, we emphasize that the ledges in the dislocation source models differ in character. Those discussed by $L i(10)$ are $C-l e d g e s$ of the type of $F i g$. ld and can each emit one edge dislocation. Those discussed by Price and Hirth ${ }^{(11)}$ are G-ledges of the type of Fig. If and involve the emission of a lattice screw dislocation together with a compensating screw GBD.

\section{VIRTUAL DISLOCATIONS}

Marcinkowski(15-18) introduced the concept of virtual dislocations, and specifically VGBD's(15). Later(19) he recognized that some of the entities, discussed in the following section, that were so described were true GBD's and dropped the antecedant virtual. However, some of his examples involve entities without true Burgers vectors so that the retention of VGBD for these would appear to have merit.

A clear example of the concept of virtual dislocations is found in the transition of an interphase interface from coherent to semicoherent, Fig. 4. In Fig. 4b, two semi-infinite crystals of different lattice parameters have been joined coherently by elastically straining the two crystals in plane strain(20). The elastic fields in the two crystals correspond to those produced by a continuous distribution of infinitesimal dislocations at the interface (21). This is proved straightforwardly by taking the limit of the strain field of a discrete array of Volterradislocations (22) as 
their Burgers vectors. $\vec{b}^{\prime}$ approach zero while their number increases inversely with $\vec{b}^{\prime}$ : the result is the requisite homogeneous strain field $(20)$. In a finite crystal, the image relaxations can also be described by the image field of the infinitesimal dislocations (21), Fig. 4c. In either case the elastic strain field can be removed, except over distances from the boundary of the order of the misfit dislocation spacing, by the introduction of true, misfit dislocations of opposite sign, Fig. 4d.

In this example, the infinitesimal, imaginary dislocations are used as Green's function generators of the appropriate elastic fields. Because these elastic fields contain only "good" material in the context of Frank's definition (23), it follows that the infinitesimal dislocations have no true Burgers vectors. Hence, it would be natural to term these defects as virtual dislocations. Moreover, if one were interested only in the long range elastic field, the same result would be achieved by replacing the array of infinitesimal dislocations by an array of imaginary dislocations with discrete lattice Burgers vectors: the result would be in error only within a distance from the boundary roughly equal to the spacing between the latter dislocations. These too would naturally be called virtual dislocations. Marcinkowski has presented the discrete dislocation analog to Fig. 4, e.g., Fig. 1 in Ref. 15. We note that he only introduced the virtual dislocations in the analog of Fig. $4 \mathrm{c}$, whereas, as indicated here, they should be introduced in either infinitesimal or discrete form in the analog of Fig. 4b. Other similar examples exist. The stress field of a crack, Mode I, II or III, in an elastically strained material can be described in terms of a continuous array of infinitesimal dislocations $(24,25)$. These cases, and other similar ones using the Dugdale-Barenblatt approach $(26,27)$, correspond to virtual dislocation problems in the sense described above. Contrariwise, there are cases where infinitesimal dislocations have true Burgers vectors. One case is the Peierls dislocation where the sum of the infinitesimal Burgers vectors equals a lattice Burgers vector, both being defined in the Burgers circuit sense as true dislocations. Similarly, when an incoherent 
precipitate forms on a dislocation, the dislocation is smeared out at the interface into a continuous distribution of infinitesimal dislocations which remain true dislocations.

\section{GRAIN BOUNDARY DISLOCATIONS AND \\ VIRTUAL GRAIN BOUNDARY DISLOCATIONS}

Originally, Marcinkowski (15-18) termed the defect formed when a dislocation cuts a grain boundary as a VGBD, but, as noted above, he later, correctly in our view, dropped the virtual notation(19). That such defects are true GBD's can be shown in several ways. First, consider the coherent, first-order twin boundary of Fig. 5a. The Burgers vector of the lattice dislocation differs after cutting because of the rotation of the glide plane across the boundary. Conservation of true Burgers vector in the cutting process leads to the result that a residual GBD must be left in the boundary, with Burgers vector (28)

$$
\vec{b}_{3}=\vec{b}_{1}-\vec{b}_{2} \text {. }
$$

For the fcc case $\left.(7,24), b_{3}=\frac{1}{6}<112\right\rangle$. Moreover, the GBD is associated with a G-ledge on the coherent interface, Fig. $5 b$ and together these comprise the so-called twinning dislocation (7) which has both the stress field and Burgers vector of a dislocation:*

$$
\text { In early work }(15-18) \text {, it was implied that the line defect of Fig. }
$$

$5 b$ was not a true dislocation but a VGBD with a long range stress field and that subsequentiy a GBD might glide in to annihilate the VGBD. In order to emphasize that the defect is a true GBD, we construct a circuit for the defect in the sense suggested by Frank ${ }^{(23)}$. In the course of the present work we will suggest two different types of circuits. The first of these, which is now described, will be called a Frank circuit. Frank(23) and Read $(29)$ have emphasized that such a circuit must begin and end on the boundary. We present the Frank circuit in the local Burgers vector sense ${ }^{(30)}$,

* This result is further verified by a careful consideration of the offsets and displacements which occur in the boundary as a result of the cutting. 
and note that it is convenient to construct separate portions of the circuit in the two crystals making up the twin. The procedure is as follows:

(a) Consider first a perfect reference twin with a planar interface as in Fig. $6 a$.

(b) In Crystal 1 of this reference system, construct a clockwise portion of the circuit, relative to the sense vector $\vec{\xi}$, lying in good material, and beginning and ending on the planar twin boundary at $S_{1}$ and $F_{1}$, respectively.

(c) In the other Crystal 2 construct a counterclockwise portion of the circuit, relative to the sense vector $\vec{\xi}$, lying in good material, beginning at the boundary at $S_{2}$, coincident with $S_{1}$, and ending at $F_{2}$, coincident with $F_{1}$.

(d) Construct the same circuit in the real system containing the GBD as in Fig. $6 \mathrm{~b}$.

(e) The closure failure from $F_{2}$ to $F_{1}$ is the Burgers vector of the dislocation in the "start to finish - right hand" ( $S F / R H)$ sense.

Hence, the dislocation in Fig. $6 b$ is the Shockley twinning dislocation with Burgers vector $1 / 6<112>$, with its stress field evident in Fig. $6 b$.

We now extend the above Frank-type definition and note that a somewhat more elegant but equivalent circuit construction can be carried out in the DSC-lattice of Bollmann in order to obtain the Burgers vector of this GBD. This DSC-lattice construction, for example, eliminates any ambiguity which might be associated with carrying out the last step across the twinning plane leading to $F_{2}$ in Fig. 6b. The twinned crystals are in a high coincidence site density misorientation, and as pointed out by Bollmann $(31)$ a coincidence lattice may be constructed (Fig. 7a) which defines all the points where the lattice points of Crystals 1 and 2 coincide; if we imagine that crystals 1 and 2 are extended throughout all of space. A DSC-lattice may also be constructed which defines all the vector displacements of the two crystals relative to one another which are possible under 
the condition that the atomic "pattern" produced by the atoms of the two crystals throughout the coincidence lattice remains unchanged. This DSC-lattice, therefore, defines all the possible Burgers vectors of both lattice and twin boundary dislocations. A section of the DSC-lattice in the common (110) plane is shown for the perfect twin boundary in Fig. 7a. The same boundary after the introduction of the twinning dislocation is shown in Fig. $7 b$. Now the DSC-lattice possesses an obvious edge dislocation whose Burgers vector is easily obtained by carrying out straightforward equivalent Frank circuits in the DSC-lattice in the dislocated bicrystal (Fig. 7b) and the perfect reference twin (Fig. 7a). We note that in this construction the GBD appears clearly as a dislocation in the DSC-lattice, and it is not even necessary to start the Frank circuit on the boundary. Evidently, the twinning dislocation (G-ledge) could be fabricated as in Figs. 1e,f by producing a monolayer step on the (111) planes of each crystal and then making the join with an accompanying displacement parallel to the twin boundary corresponding to the Burgers vector.

The twinning dislocation corresponding to the monolayer G-ledge in Figs. $5 b, 6 b$ and $7 b$ clearly has a long range stress field. We now show that a corresponding $G-l e d g e$ which is three monolayers high may dissociate into a lattice dislocation and a pure ledge which possesses no long range stress field. We imagine that three identical twinning dislocations of the type shown in Fig. 7b on successive [111] planes coalesce, and that a G-ledge is produced which is three units high and possesses a Burgers vector $3 \vec{b}_{3}$. How ever, the vector $3 \vec{b}_{3}$ is seen to be a lattice vector. In this case the $G$ ledge can dissociate into a lattice dislocation and a pure ledge of the type shown in Fig. 7c. (Alternatively a dislocation with Burgers vector $-3 \vec{b}_{3}$ can run into the array and produce the pure ledge). A Frank circuit in the DSClattice, around the ledge (Fig. 7c) exhibits closure in this case when constructed in the reference lattice of $\mathrm{Fig}$. $7 \mathrm{a}$ as can be seen by inspection. of course, this pure ledge could be fabricated as in figs. 1e,f by producing matching trilayer steps on the two crystals in fig. le and then making the 
join as in Fig. If without any further relative displacements.

Figure 7b illustrates the determination of a GBD Burgers vector of the type $1 / 3<111>$ lying normal to the twin boundary. Together with the example of Fig. 7b with its Burgers vector parallel to the boundary, the latter example generalizes the method.

The above definition of GBD's is consonant with the conventional view of partial dislocations. The GBD in Fig. 7b corresponds to the partial twinning dislocation, for example, as formed by the dissociation of perfect dislocations in the fcc lattice, and that of Fig. $7 d$ is a Frank partial dislocation.

\section{IMPOTENT GRAIN BOUNDARY DISLOCATIONS}

We now draw attention to the fact that the pure ledge in Fig. 7c. can be considered to have dislocation character in the same way that Read(29) has shown that grain boundaries do. While the Frank circuit construction does not reveal this dislocation character, a circuit can be developed to reveal it as discussed below. Since the dislocations which are responsible for the dislocation character associated with this pure ledge have no long range stress field, we call them impotent grain boundary dislocations (IGBD's). to distinguish them from the GBD's as defined in the preceding section.

In order to describe the IGBD's, consider Fig. 8a which represents the coherent twin boundary itself as a tilt boundary with one twinning dislocation of the type $\frac{1}{6}<112>$ per atom plane cut by the boundary $(32,33)$. (A) further demonstration of this dislocation representation is given in Fig. 9a.)*

* We remark that such a twin boundary could be built up by accumulating a stack of twinning dislocations by running them together by glide on succassive (111) type planes. There is a subtle point with regard to the equivalency of this configuration with the twin structure in Figs. 8 a and 9 a that has not been explicitly discussed in the literature. Motion of twinning dislocations in the direction $n$, on the twinning plane $k$, for the common boat-shaped twin naturally produces the requisite rotation of the undistorted plane $K_{2}$ into the twin orientation. For such a twin, operation of twinning dislocations of the conjugate system $n_{2}$ on plane $K_{2}$ appears not to give the necessary rotation (see Fig. 23-2b in Ref. 33). However, one must realize. that the closely spaced twinning dislocations comprise a tilt boundary as in Figs. 8 a and $9 a$, the displacement field of which produces the requisite rotation. One can think of the boundary as a wedge driven into the crystal 
The final configuration of Fig. 8 can be generated by superposing a set of dislocation dipoles as in Fig. 8a. This set has no long range strain field: it is the impotent distribution of dislocations defined by Mura $(34,35)$. To illustrate this fact we demonstrate that the dislocation dipole arrays $A B$ and $C D$ produce identically equal and opposite shear displacements so that together they produce a rotation but no long range elastic strain. The three dislocations with Burgers vectors $\frac{1}{6}[\overline{1} \overline{1} 2]$ in $A B$, moving over a distance $L$ on $p l a n e$ (111), produce a shear per unit length of dislocation in a unit volume given by

$$
\varepsilon=3 \mathrm{bL} \text {. }
$$

The dislocations in $C D$ with Burgers vectors $\frac{1}{6}$ [112] move over a distance $h$ on (11i). There are $N$ of them in the length $L$, so they produce a shear displacement in the opposite sense of $A B$,

$$
\varepsilon=b h N \text {. }
$$

However, since the glide planes are inclined at the angle $\theta$ between (111) and (iii), $h=3 d / \sin \theta$ and $N=L \sin \theta / d$, where $d$ is the interplanar spacing of \{111\} planes. Thus, equations (2) and (3) are equal and opposite.

We now suggest a circuit for the determination of the Burgers vectors of IGBD's. Since this is analogous. to the scheme presented by $\operatorname{Read}(29)$ for the determination of the dislocation content of a grain boundary, and because we wish to distinguish it from the other circuits, we call it a Read circuit. For clarity we consider the same example as that of Fig. 7. Each crystal is now defined by a different lattice, corresponding to a rotated DSC-lattice as shown in Fig. 9a. The perfect reference lattice is the corresponding DSC-lattice in a perfect, untwinned crystal. As can be seen by comparison of Figs. $9 a$ and $7 a$, the perfect reference DSC-lattice is identical to the DSC-lattice drawn in Fig. $7 a$, and hence it is not redrawn separately.

Footnote continued from preceding page:

* which produces the rotation (indeed this operation corresponds to motion of a wedge disclination into the crystal). In the sense that the rotation accompanies the motion of the twinning dislocations, the latter do not "belong" to either. of the crystals adjoining the boundary but are symmetrically disposed with respect to both crystals. 
The Read circuit, defined as before, is also depicted in Fig. $9 a$. The net Burgers vector of the IGBD's in the boundary is given by the vector connecting $F_{2}$ to $F_{1}$ as before. This vector can be interpreted in two ways, both of which are represented in $F i g$ : $9 a$. Decomposed into component vectors $F_{2} G$ and GF 1 in the rotated DSC-lattices the decomposed Burgers vectors each contain . three IGBD's of the $\frac{1}{6}<112>$ type, the sum of which, six, corresponds to the six planes ending on the section of tilt boundary shown on the figure. However, the net Burgers vector can also be represented in the common DSC-lattice of the two crystals, also presented in Fig. 9a (shown dashed). In that common-DSC-lattice the net Burgers vector of the IGBD's is the sum of those of four IGBD's of the type $\frac{1}{3}<111>$ all of which are normal to the twin boundary. The latter representation corresponds to the grain boundary resultant Burgers vector as defined in formal grain boundary theory $(36,37,38)$.

Figure $9 b$ presents the Read circuit equivalent of Fig. $7 b$. As can be, seen by comparison, the net Burgers vector includes the Shockley partial revealed by Fig. 76 but also includes the IGBD content of the twin. The net IGBD Burgers vector is given, in general, by the difference between the net Burgers vector content of Frank and Read circuits which are identical in the perfect reference DSC-lattice and which start at the same point on the twin boundary in the real system. Thus, both circuits are necessary for a complete analysis of a given boundary.

The Frank partial GBD case is reanalyzed in Fig. 9c. Again both the GBD and the IGBD content are revealed in the Read circuit of Fig. $9 \mathrm{c}$. The pure ledge case is reanalyzed in Fig. $9 d$. The IGBD content of this system, including the IGBD's of the ledge itself, is determined by the circuit.

In order to illustrate the generality. of the result we now consider a higher order twin plane, Fig. 10, the $\{210\}$ twin in a simple cubic lattice. Fig. 10 shows the twin with a pure ledge containing sixIGBD's with Burgers vector $\frac{1}{5}\langle 210\rangle$. The closure failure in a Read circuit as specified above, and as shown in Fig. 10, gives the net Burgers vector. That the configuration corresponds to a stress-free pure ledge in this case is evi- 
dent from the crystal arrays. To emphasize that arrays such as those in Fig. 10 can be produced from a perfect twin by the superposition of an impotent array of dislocations $(34,35)$, we show an impotent array for this case in a corner of Fig. 10. Clearly, this array produces rotation but has no long range stress field: also its superposition on a planar twin boundary produces two pure ledges, while its superposition on the pure ledge of fig. 10 produces translation of the latter.

IGBD's in pure ledges obviously can be considered as superpositions of GBD's as illustrated by the process of creation of Fig. 7c, which can be formed by three GBD's of the type of Fig. 7b superposed on a perfect lattice dislocation, a mechanism discussed previously:

One can also regard some IGBD's as the superposition of a GBD and a VGBD. For illustration of this point, suppose that the dislocation array E representing part of the twin boundary of Fig. 8 was displaced as in Fig. 3c, without cutting by lattice dislocations. This produces a nonequilibrium configuration with a long range stress field. The displacement is equivalent to superposition of dipole dislocations $C D$ in the absence of $A B$. As discussed above, this superposition is equivalent to a shear without the accompanying rotation. In the context of the previous footnote, the situation would be analogous to motion of the conjugate twinning dislocations without rotation, which would not produce the equilibrium boundary. As we have already shown, dipole dislocations $A B$ have a strain. field equal and opposite to that of $C D$. Hence, the configuration in Fig. $8 \mathrm{c}$ has the stress field of a dislocation and a Burgers vector as defined by a Frank circuit, but is not created in the usual cut-and-displacement method of Volterra dislocations. Hence, the defect could logically be called a VGBD. Superposition of the GBD A upon this VGBD reproduces the equilibrium long-range stress free configuration, i.e., the IGBD of Fig. 8b. Thus, configurations such as Fig. 6b in Ref. 15 do show VGBD's which have the properties discussed there, but only if the boundary is initially displaced without the plastic shear strain produced oy cutting dislocations, not with the plastic shear as in 
Ref. 15 .

We note that the operation of Fig. $8 \mathrm{c}$ may be most easily envisioned for a small angle boundary. There is a discrete, attractive, elastic interaction energy between arrays $E$ and $F$ which would tend to restore the perfect tilt boundary configuration. Hence, the depicted displacement of array $F$ requires work, done by an external stress, say. If displaced reversibly, this work is exactly that to create the stress field of the VGBD created in Fig. $8 c$.

0 ther VGBD configurations can be produced readily. For example, Fig. 11 illustrates an initially perfect low angle tilt boundary dissociating into a boundary containing a VGBD and a perfect lattice dislocation. Alternatively, this array could obviously be formed by superposing a true lattice dislocation on one of the IGBD's comprising the tilt boundary.

The above discussion has focused on the cases of twin boundaries and low angle boundaries. In the next section it is shown to apply in general to grain boundaries.

\section{SECONDARY GBD's}

Many of the above concepts can be extended directly to the description of secondary GBD's in boundaries having a misorientation near a coincidence lattice $(6,3.1,39-43)$ misorientation and connected with the formal theory of GBD's(31). In terms of coincidence lattice theory the coherent twin boundary of the previous section is the symmetric $\Sigma=3$ boundary (i.e., one in three atoms belongs to the coincidence lattice as in Fig. 7a) for rotation about $\langle 110\rangle$ in the fcc structure, and the boundary in Fig. 10 is the $\Sigma=5$ boundary for rotation about $\langle 100\rangle$ in the simple cubic lattice.

In order to show the extension explicitly, let us treat the special class of symmetrical tilt boundaries obtained in the simple cubic system by rotating each crystal around [001] away, from its original common orientation by the angle given by $\tan ^{-1}(1 / n)$, where $n=$ integer $=$ Miller index of the boundary plane relative to either crystal $(n 10)$. There are then a succession of coincidence lattices (and boundaries) with lower and lower degrees of co- 
incidence as denoted in Table $1 .{ }^{*}$ As we have already pointed out, a series of corresponding DSC-lattices (31) may then be constructed which define all of the possible Burgers vectors of the GBD's which may be present in such boundaries under the condition that a similar coincidence configuration is present in the boundary on either side of the GBD. Simple geometric demonstrations of the above statement have been presented by Balluffi, et al ${ }^{(6)}$. When the above operations are applied for coincident lattice situations with $\Sigma>1$, the dislocations are often called secondary GBD's ${ }^{(31)}$. Also listed in Table 1 are the Burgers vectors of those of the secondary GBD's which are of edge type and lie parallel to the [001] tilt axis. Secondary IGBD's, and VGBD's are defined in an analogous way.

Now we show that for a small angle boundary, the GBD's formed by the analog, Fig. 12, of the cutting process previously presented in Section 4 are identically the secondary GBD's predicted by the formal theory. For the low angle tilt boundary in the simple cubic lattice of Fig. 12a, the spacing $D$ between IGBD's is given by $(23,41)$

$$
b / D=2 \sin (\theta / 2) \text {. }
$$

With this inclination of glide planes, the GBD left at the boundary, Fig. 12, has a Burgers vector of magnitude

$$
b_{3}=2 b \sin (\theta / 2)=b^{2} / 0 \text {. }
$$

Also, a regularly spaced tilt boundary corresponds to lattice coincidence over each interval $h$ as shown in Fig. 12c, where $h=2 D$. The geometry of Fig. $12 c$ indicates that

$$
h^{2}=(2 D)^{2}=b^{2}+(n b)^{2} .
$$

Substituting equation (6) into equation (5) we find

$$
b_{3}=2 b /\left(1+n^{2}\right)^{\frac{1}{2}}
$$

which is identically the Burgers vector of one or a pair of secondary IGBD's for the (n10) coincidence boundary, Table 1 . Thus on cutting a small angle

* We note that we have adopted this special class of boundaries for purposes of simplicity and that it does not include all possible symmetric [001] tilt coincidence boundaries in the simple cubic system. 
boundary, the resulting $G B D$ is a secondary GBD.

Now consider a tilt boundary between crystals with the same relative rotations but for which the boundary plane is rotated with respect to the above example so that it contains ledges, which, in turn contain IGBD's. Between the ledges, the boundary is identical to the above case so the situation on cutting it is the same as in the above case.

Finally, consider an irregular tilt boundary, where the boundary is a true grain boundary, i.e., a boundary without any long range stress field, but where the dislocation spacing, although periodic, is not unform. In general, such a boundary can be decomposed into two or more superposed regular tilt boundaries with uniform dislocation spacings(29). Specifically, suppose that the boundary of Fig. 12a has superposed upon it a regular array with spacing $h^{\prime}=n h$, where $n$ is an integer considerably larger than unity. This array no longer has the same coincidence lattice or DSC-lattice as that of Fig. 12. Because of the rotation accompanying the introduction of the new dislocations, a new coincidence lattice (higher order in this case) with a different DSC-lattice (smaller in this case) will be formed. Now on cutting, even of a segment that resembles the array of Fig. 12 , the secondary GBD formed will be a new GBD corresponding to the new DSC-lattice.

The above results may be generalized by realizing that the DSClattice, which contains all possible Burgers vectors of the secondary GBD's, also contains all the various Burgers vectors of the lattice dislocations in the two grains adjoining the boundary. This statement follows from the fact that the lattice vectors are also pattern-conserving displacement vectors. Therefore, all lattice Burgers vectors, and all differences between the various lattice Burgers vectors, must be expressible in terms of integral combinations of the various secondary GBD Burgers vectors. Thus, the GBD's produced in the boundary by any cutting process by lattice dislocations must be secondary GBD's. In addition, any lattice dislocation which impinges upon the boundary can decompose into an integral number of secondary GBD's. A specific demonstration of this latter phenomenon has been given elsewhere ${ }^{(43)}$. 
Frank $(36)$ has suggested that any grain boundary can be formally regarded as the superposition of arrays of perfect lattice dislocations, i.e., primary IGBD's. In view of the above developments, this postulate can be generalized in that any grain boundary can be formally regarded as the superposition of arrays of primary IGBD's and secondary IGBD's. * Secondary IGBD arrays would possess the DSC-lattice vectors $(31)$ as Burgers vectors for crystals with arbitrary degrees of coincidence. Recursion formulas such as those in Table 1 can be developed readily for other types of coincidence relations and in other crystal structures, e.g., see Ref. 41,44 . In all cases, $|\vec{b}|$ falls off approximately as $1 / \sqrt{\Sigma}$, and in the limit of very high order secondary GBD's, the dislocations become infinitesimal. Bollmann(45) prefers to term secondary dislocations only those corresponding to relatively low $\Sigma$ interfaces which might, for example, be observed in transmission electron microscopy (31,41-43). However, what one might call the extended secondary dislocations of large $\sum$ as defined above belong to the generalized DSC-lattice of the two grains and have all of the formal properties of GBD's and hence can properly be called secondary GBD's. Of course, for say very large $\Sigma$, or, equivalently, very small $|\vec{b}|$, one would not expect to detect the individual GBD's by transmission electron microscopy or other physical means, so the extension. is strictly a formal one. ${ }^{\dagger}$

The continuous surface dislocation model $(46)$ describes boundaries in terms of a continuous distribution of infinitesimal dislocations in the boundary. Evidently from the above discussion, such a boundary can be composed of infinitesimal dislocations which are secondary IGBD's as defined above, so the two models would then be identical. Thus, the extended secondary GBD model provides a connection between the Frank ${ }^{(36)}$, Bollmann ${ }^{(31)}$, and surface dislocation $(46)$ models.

* The secondary GBD's and secondary IGBD!s of the present work are respectively the "extrinsic" and "intrinsic" GBD's of Balluffi, et al. in Ref. 6.

$+A$ discussion of the range of conditions over which the formal secondary IGBD model for high angle grain boundaries may be expected to constitute a physically satisfactory model has been given recently by R. W. Balluffi and T. Y. Tan, Materials Science Center Report \#1829, Cornell University, Ithaca, New York (to be published, Scripta Met.). 
Also of interest is the minimum IGBD or GBD Burgers vector for a given boundary. In a simple cubic lattice, specifically for a (410) coincidence boundary, secondary dislocations with $\vec{b}=(1 / 17)[410]$ are listed in Table 1 . These could in principle dissociate by reactions such as

$$
\frac{1}{17}[400]=\frac{1}{34}[350]+\frac{1}{34}[530]
$$

or other further splittings. However, these would essentially be core-type relaxations and would not change the analysis. For boundaries which contain ledges a plausible choice for a minimum Burgers vector IGBD would be that for which there is one IGBD per ledge of monatomic height, since further dissociations can be regarded as core relaxations as mentioned above.

This interrelation is of interest with respect to configurations such as that in Fig. 13. While there would be no long-range elastic interaction, the short-range interaction between the IGBD or pure ledge pairs $A$ and $B$ would be a dipole-dipole type interaction according to section 5 , and thus either configuration could be stable. This result is of applicability to the problem of the faceting of grain boundaries.

\section{ADDITIONAL REMARKS AND SUMMARY}

Our discussion throughout has focussed on grain boundary line defects with the aim of describing them systematically and developing a consistent terminology for them. Some dislocation arrays can be regarded alternatively as disclinations: for example, a tilt wall of dislocations terminating in a lattice corresponds to a wedge disclination. A discussion of some of the physical entities treated here in terms of disclinations has been developed by Sadananda, et al (47). For example, the VGBD of Fig. $8 \mathrm{c}$ is the wedge disclination dipole discussed by the latter authors.

Finally, we present in Table 2 a brief summary of the major types of grain boundary line defects which have been described in the present paper.

\section{ACKNOWLEDGEMENT}

We wish to thank Drs. M. J. Marcinkowski and W. Bollmann for useful discussions. 
1. H. Gleiter and G. Bäro, Mat. Sci. Eng. 2, 224 (1967).

2. H. Gleiter, E. Hornbogen and G. Bäro, Acta Met. 16, 1053 (1968).

3. G. Bäro, H. Gleiter and E. Hornbogen, Mat. Sci..Eng. 3 , 92 (1968).

4. T. Schober and R. W. Balluffi, Phil. Mag. 24, 469 (1971).

5. M. F. Ashby, Scripta Met. $\underline{3}, 843$ (1969):

6. R. W. Balluffi, Y. Komem and T. Schober, Surf. Sci. 31, 68 (1972).

7. A. W. Sleeswyk and C. A. Verbraak, Acta Met. 9 , 917 (1961).

8. C. Laird and H. I. Aaronson, J. Inst. Met. $\underline{96}, 222$ (1968).

9. W. K. Burton, N. Cabrera and F. C. Frank, Phil. Trans. Roy. Soc. (London) 243, 299 (1951).

10. J.C.M. Li, Trans. AIME 227, 239 (1963).

11. C. W. Price and J. P. Hirth, Mat. Sci. Eng. 9, 15 (1972).

12. C. Herring, in the Physics of Powder Metallurgy, p. 143, McGraw-Hill, New York. (1951).

13. F. C. Frank, in Symposium on Plastic Deformation of Crystalline Solids, p. 89, Carnegie Institute of Technology, Pittsburgh (1950).

14. J. P. Hirth, in Relations between Structure and Strength in Metals and Alloys, p: $218, H$. M. Stationery Office, London (1963).

15. M. J. Marcinkowski, in Fundamental Aspects of Dislocation Theory (eds. J. A. Simmons, R. dewit and R. Bullough) P. 531, N.B.S. Spec. Publ. 3i7, Washington, D. C. (1970).

16. M. J. Marcinkowski and W. F. Tseng, Met. Trans. 1,3397 (1970).

17. M. J. Marcinkowski, in Proc. 5 th International Materials Symposium, Sept. 1971.

18. E.S.P. Das and M. J. Marcinkowski, J. Appl. Phys. 42, 4107 (1971).

19. E.S.P. Das and M. J. Marcinkowski, Acta Met. 20, 199 (1972).

20. J. S. Vermaak and J. H. Van der Merwe, Phil. Mag. 12, 453 (1965).

21. K. Shinohara and J. P. Hirth, Phil. Mag., in press.

22. C. S. Hartley, Scripta Met. 3,607 (1969).

23. F. C. Frank, Phil. Mag. 42, 809 (1951).

24. J.P. Hirth and J. Lothe, Theory of Dislocations, McGraw-Hill, New York (1968).

25. J. R. Rice, Fracture, Vol. II, p. 191, Academic Press, New York (1968).

26. D. S. Dugdale, J. Mech. Phys. Sol. 8,100 (1960). 
27. G. I. Barenblatt, Adv. Appi: Mech. $\underline{7}, 1$ (1962).

28. J. Friedel, Dislocations, Addison-Wesley, Reading, Mass. (1964).

29. W. T. Read, Jr., Dislocations in Crystals, McGraw-Hill, New York (1953).

30. Ref. 24, p. 21 .

31. W. Bollmann, Crystal Defects and Crystalline Interfaces, SpringerVerlag, New York (1970).

32. R. Bullough, Proc. Roy. Soc. (London) A241, 568 (1957).

33. Ref. 24, p. 741 .

34. T. Mura, Europhysics Conference on Disclinations, Aussois, France, June 1972 .

35. T. Mura, in Mechanics of Generalized Continua (ed. E. Kröner) p. 269, Springer-Verlag, Berlin (1968).

36. F. C. Frank, in Report of the Symposium on the Plastic Deformation of Crystalline Solids, p. 150, Carnegie Institute of Technology, Pitts burgh $(1950)$.

37. Ref. 24, p. 648 .

38. R. Bullough, Phil. Mag. 12, 1139 (1965).

39. D. G. Brandon, Acta Met. 14, 1479 (1966).

40. G. H. Bishop and B. Chalmers, Phil. Mag. 24, 515 (1971).

41. T. Schober and R. W. Balluffi, Phil. Mag. 21, 109 (1970).

42. T. Schober and R. W. Balluffi, Phys. Stat. Sol. (b) 44, 115 (1971).

43. T. Schober and R. W. Balluffi, Phil. Mag. 24, 165 (1971).

44. T. Schober, Phil. Mag. 22, 1063 (1970).

45. W. Bollmann, private communication, December, 1971.

46. B. A. Bilby, R. Bullough and E. Smith, Proc. Roy. Soc. (London) A231, $263(1955)$.

47. K. Sadananda, E.S.P. Das and M. J. Marcinkowski, Univ. of Maryland Rept. E.M.G. 85; Met. Trans., in press. 
TABLE 1: Symmetrical coincidence boundaries produced by rotation about [001] of $\theta=2 \tan ^{-1}(1 / n)$ in a simple cubic lattice. The angle of rotation $\theta$, reciprocal coincidence density $\Sigma$, secondary dislocation Burgers vectors of a portion of the DSC-lattice, and their magnitudes are given. Units of $|\vec{b}|$ are lattice parameters. The $n$th $A$ set is for $n$ even; the $B$ set for $n$ odd.

\begin{tabular}{|c|c|c|c|c|}
\hline $\begin{array}{l}\text { Boundary } \\
\text { Plane }\end{array}$ & Radians & $\Sigma$ & $\vec{b}$ & $|\vec{b}|$ \\
\hline$(210)$ & 0.92 & 5 & $(1 / 5)[210]$ & $1 / \sqrt{5}$ \\
\hline$(310)$ & 0.64 & 5 & $(.1 / 5)[310]$ & $2 / \sqrt{10}$ \\
\hline$(410)$ & 0.50 & 17 & $(1 / 17)[410]$ & $1 / \sqrt{17}$ \\
\hline$(510)$ & 0.39 & 13 & $(1 / 13)[510]$ & $2 / \sqrt{26}$ \\
\hline$(610)$ & 0.33 & 37 & $(1 / 37)[610]$ & $1 / \sqrt{37}$ \\
\hline$(710)$ & 0.29 & 25 & $(1 / 25)[710]$ & $2 / \sqrt{50}$ \\
\hline : & : & : & $:$ & : \\
\hline$(n 10)_{A}$ & $2 / n$ & $n^{2}+1$ & $\frac{1}{n^{2}+1}[n 10]$ & $1 / \sqrt{\left(n^{2}+1\right.}$ \\
\hline$(n 10)_{B}$ & $2 / n$ & $\frac{n^{2}+1}{2}$ & $\frac{2}{n^{2}+1}[n 10]$ & $2 / \sqrt{n^{2}+1}$ \\
\hline
\end{tabular}


TABLE 2: Summary of major types of grain boundary line defects and circuits for revealing them.

\begin{tabular}{|c|c|}
\hline Entity & Description \\
\hline $\begin{array}{l}\text { grain boundary } \\
\text { dislocation (GBD) }\end{array}$ & $\begin{array}{l}\text { a dislocation lying in grain bound- } \\
\text { ary. Its Burgers vector is a } \\
\text { Burgers vector of the DSC-lattice. } \\
\text { It may, or may not be, associated } \\
\text { with a grain boundary ledge (see } \\
\text { below). }\end{array}$ \\
\hline climb ledge $(C-l e d g e)$ & $\begin{array}{l}\text { a ledge in grain boundary associated } \\
\text { with a GBD which requires climb to } \\
\text { move in boundary plane. }\end{array}$ \\
\hline glide ledge (G-ledge) & $\begin{array}{l}\text { a ledge in grain boundary associated } \\
\text { with a GBD which may glide in bound- } \\
\text { ary plane. }\end{array}$ \\
\hline pure ledge & $\begin{array}{l}\text { a ledge in grain boundary which is } \\
\text { not associated with a GBD. How- } \\
\text { ever, it may be associated with an } \\
\text { IGBD (see below). }\end{array}$ \\
\hline $\begin{array}{l}\text { impotent grain } \\
\text { boundary dislocation } \\
\text { (IGBD) }\end{array}$ & $\begin{array}{l}\text { a GBD in a configuration which does } \\
\text { not possess a long range stress } \\
\text { field.. }\end{array}$ \\
\hline $\begin{array}{l}\text { virtual grain boundary } \\
\text { dislocation (VGBD) }\end{array}$ & $\begin{array}{l}\text { linear entity in grain boundary } \\
\text { which has the stress field of a } \\
\text { GBD but which may be regarded as } \\
\text { not possessing a true Burgers } \\
\text { vector. }\end{array}$ \\
\hline Frank circuit & $\begin{array}{l}\text { modified Burgers-type circuit for } \\
\text { revealing GBD's. }\end{array}$ \\
\hline Read circuit. & $\begin{array}{l}\text { modified Burgers-type circuit for } \\
\text { revealing both GBD's and IGBD's. }\end{array}$ \\
\hline
\end{tabular}




\section{FIGURE CAPTIONS}

Fig. 1: (a)-(b) Construction of reference bicrystal containing a grain boundary without GBD's.

(c) -(d) Introduction of a GBD with C-ledge character into grain boundary of bicrystal shown in (b). The displacement across the cut in this case is perpendicular to the boundary, and material is removed from Crystal 1.

(e)-(f) Construction of either a pure grain boundary ledge or a GBD with G-ledge character.

Fig. 2: A ledge at a coherent interface between an A rich (hollow circles) region and $a$ rich (solid circles) region for the case where the lattice parameters of $A$ and $B$ are identical. Growth of the $B$ rich region by a series of diffusion jumps leading to ledge motion is shown by the arrows.

Fig. 3: Ledge at a coherent interface, as in Fig. 2, decomposing into a perfect interface and two lattice edge dislocations.

Fig. 4: (a) Two epitaxially oriented crystals of different lattice parameters:

(b) Crystals joined to form a coherent interface whose strain field is represented by the continuous array of infinitesimal dislocations shówn.

(c) Same as previously but with image relaxations at free surfaces.

(d) Relaxation of the long range elastic field of (b) by the introduction of interface dislocations.

Fig. 5: (a) Coherent first order twin boundary in a fcc crystal.

(b) Boundary after cutting by a perfect lattice dislocation. (Indices refer to the left crystal except for the one.with the subscript T).

Fig. 6: View parallel to the (111) twinning plane and normal to the (110) plane in a fcc crystal. 
(a) Perfect reference twin.

(b) Twin boundary containing $\frac{1}{6}$ [i $\left.\overline{1} 2\right]$ twinning dislocation.

Fig. 7: (a) First-order twin boundary in the fcc structure. Atomic positions same as in Fig. 6a. Fine lines represent the DSC-1attice. Two cells of the coincidence lattice also shown. The perfect reference twin Frank circuit corresponding to Fig. 7b is also given.

(b) Twin boundary of (a) after introduction of a Shockley twinning dislocation; i.e., a GBD with Burgers vector $\frac{1}{6}<112>$. Frank circuit is also shown.

(c) Twin boundary with pure ledge. Frank circuit is also shown.

(d) Twin boundary containing a GBD with Burgers vector normal to the boundary. Frank circuit is also shown.

Fig. 8: Generation of a boundary inclined relative to the (111).twin plane orientation. Twin boundary is represented by dislocations on the conjugate twin plane.

(a). Inclined plane is generated by the superposition of the dipole array $A B C D$.

(b) Resultant inclined configuration.

(c) Glide of a portion of the boundary without cutting.

Fig. $g$ : (a) First-order twin boundary in the fcc structure. Atomic positions identical to Fig. 7a. Lines represent lattices equal in measure to the DSC-lattice of Fig. 7 a but rotated with respect to it. Read circuit is also shown. Total Burgers vector $\vec{b}_{T}=F_{2} F_{1}$ of the circuit is shown along with its decomposition into four IGBD's with Burgers vectors $\left.\frac{1}{3}<111\right\rangle$ in the common DSC-lattice of the two crystals, shown as dashed lines. Alternate decomposition into Burgers vectors $F_{2} G$ and $G F_{1}$, each containing three IGBD's with Burgers vectors $\left.\frac{1}{6}<112\right\rangle$ in the rotated DSC-lattices is also shown.

(b) Fig. $7 b$ redisplayed in the rotated DSC-lattices with a Read 
circuit.

(c) Fig. $7 d$ redisplayed in the rotated lattices with a Read circuit.

(d) Fig. $7 c$ redisplayed in the rotated lattices with a Read circuit.

Fig. 10: A (210) twin in a simple cubic structure with a pure ledge in the boundary. Coincidence lattice is indicated by solid circles. Read circuit reveals IGBD content. Inset shows impotent array of IGBD's.

Fig. 11: Dissociation of a perfect low-angle twin boundary (a) into a VGBD and a lattice dislocation (b).

Fig. 12: (a) Low angle tilt boundary.

(b) Boundary after cutting by a perfect lattice dislocation.

(c) Unrelaxed atomic arrangement near boundary for a simple cubic crystal.

Fig. 13: Two possible local arrangements of IGBD-ledge pairs $A$ and $B$. 

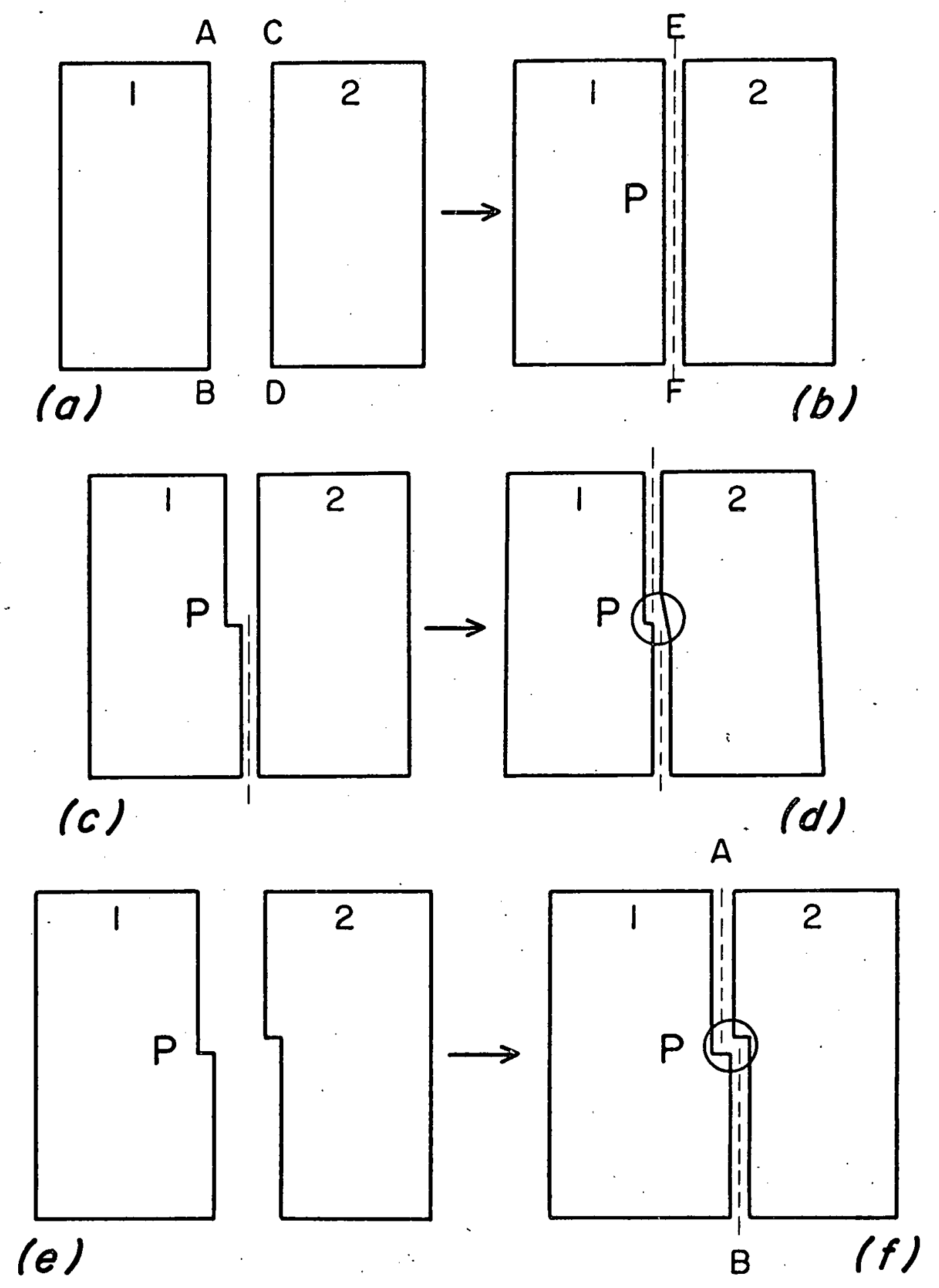

Figure 1. 
00000000

0000000000

0001000000

$0 \quad 0 \quad 0<0<0<0 \quad 0 \quad 0$

000000000

0000000000

000000000

00000000

Figure 2. 
00000000

00000000

00000000

00000000

00000000

00000000

00000000

00000000

(a)

Figure 3a. 
00000000

00000000

00000000

Q OOOOOOOO

00000000

00000000

00000000

00000000

(b)

Figure 3b. 

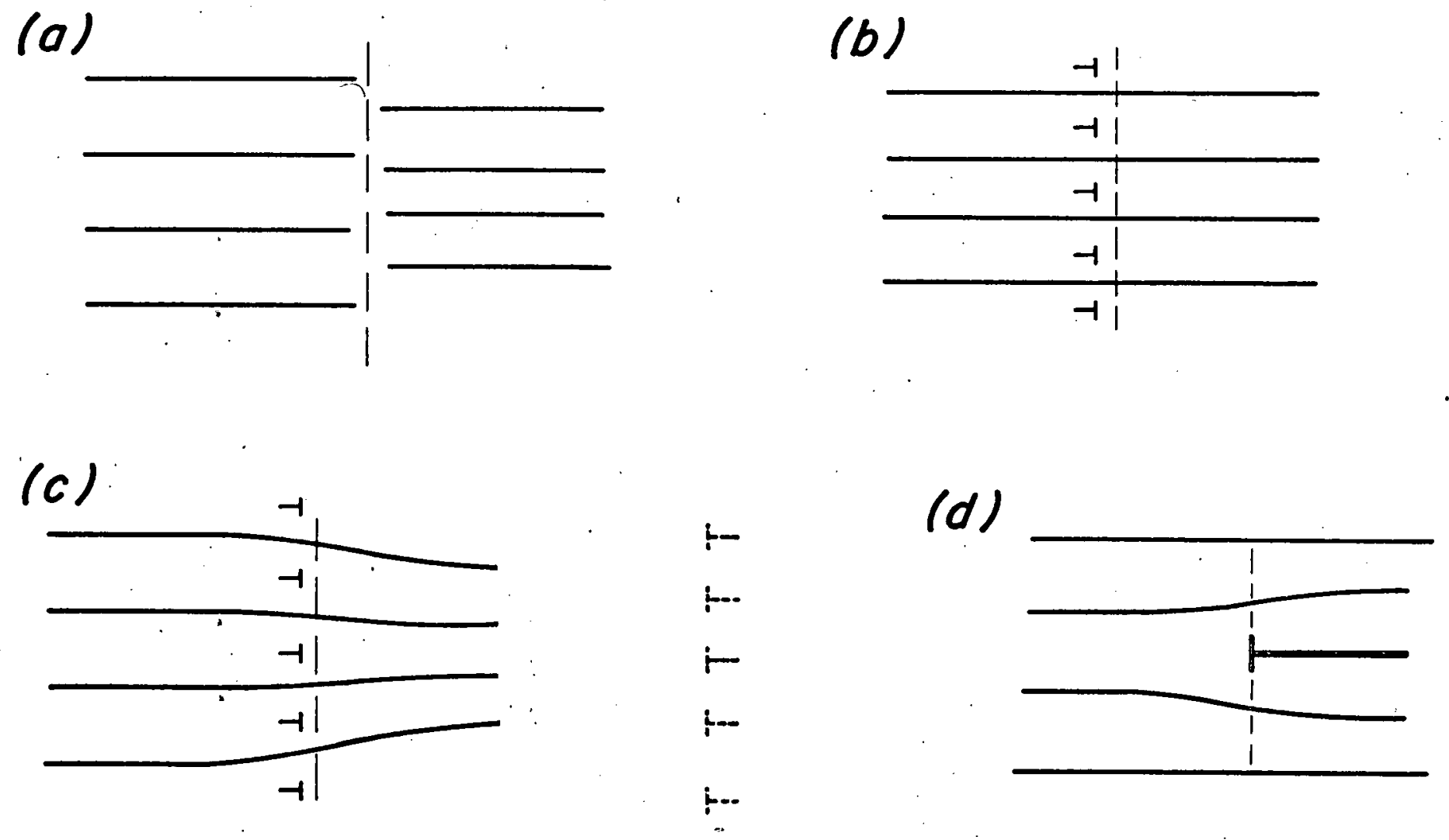
(a)

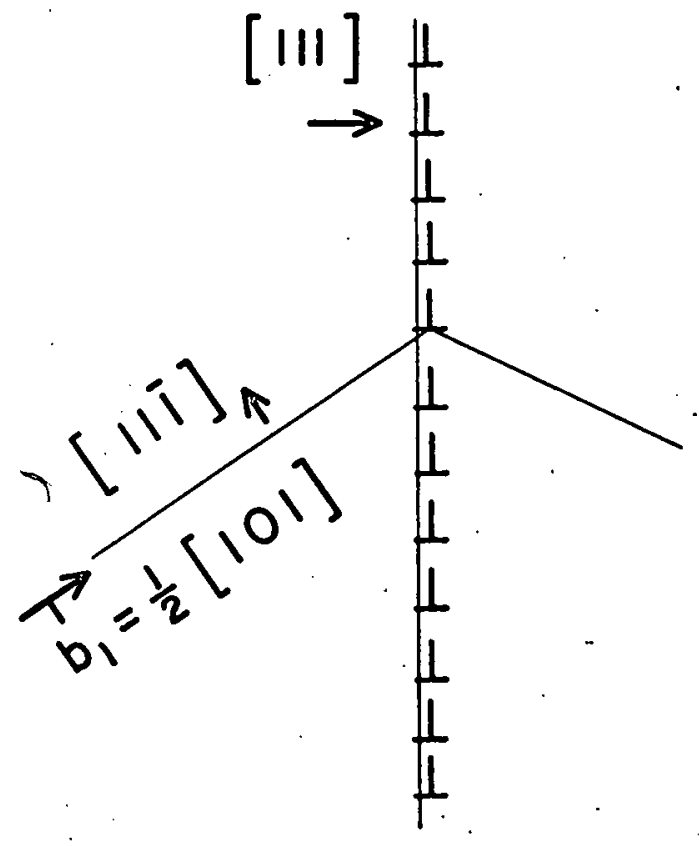

(b)

$\frac{\mathbb{N}}{\frac{1}{H}} \uparrow b_{3}=\frac{1}{6}[i \tau 2]$

$\vdash$

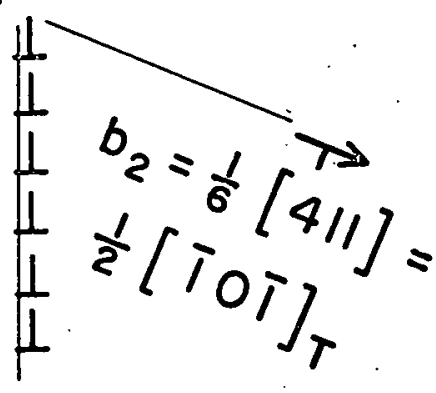

Figure 5. 


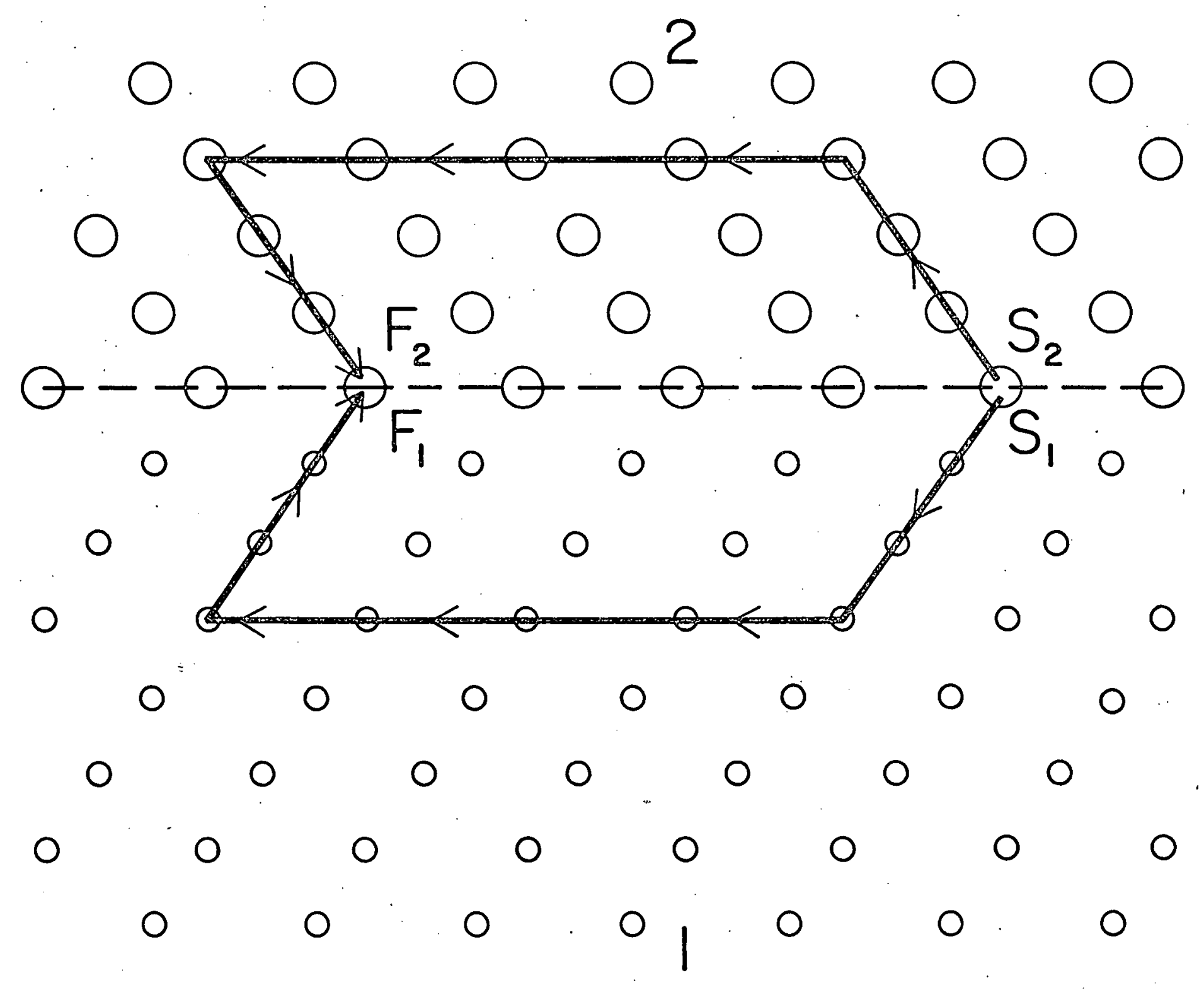



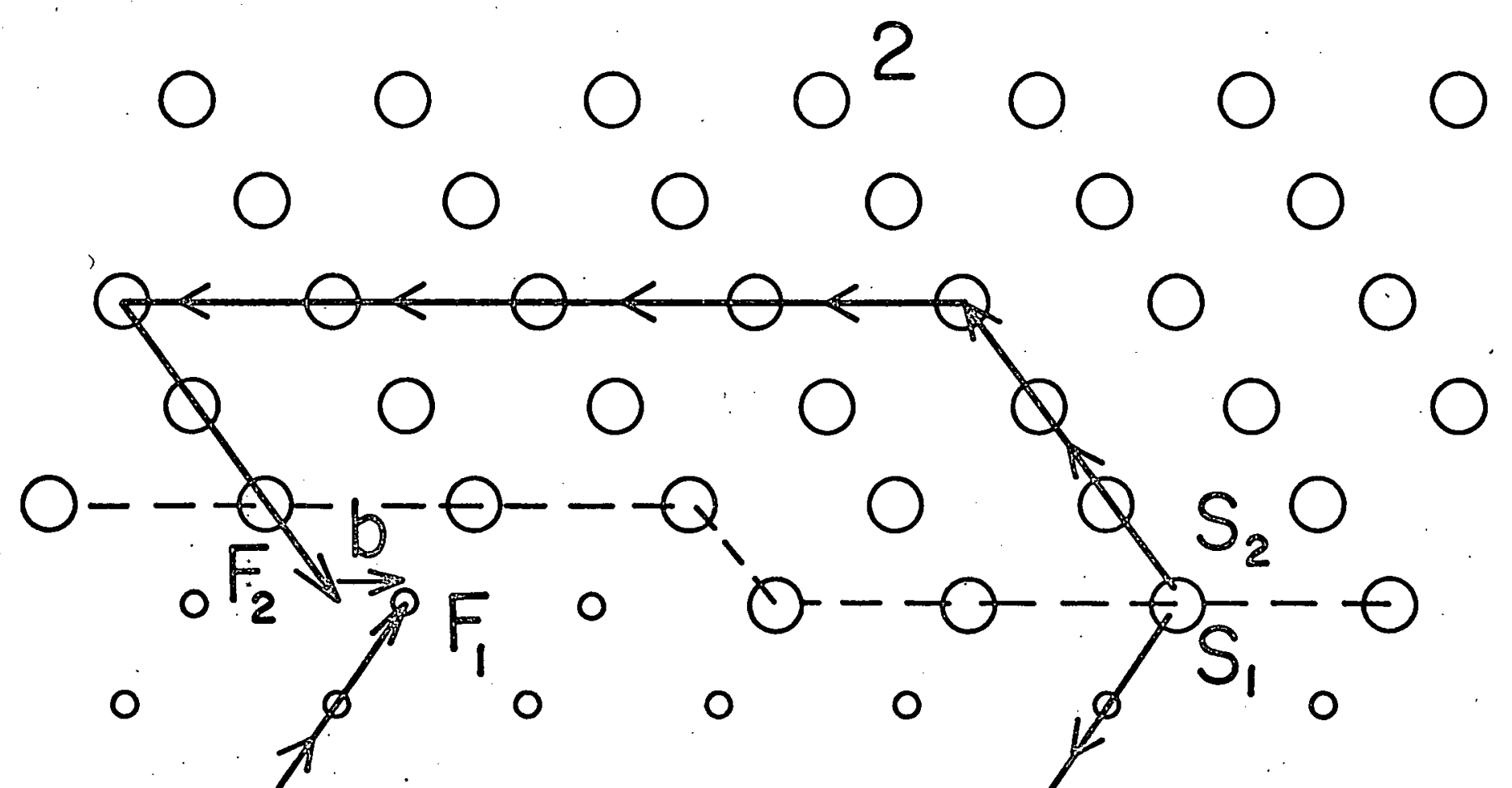

0
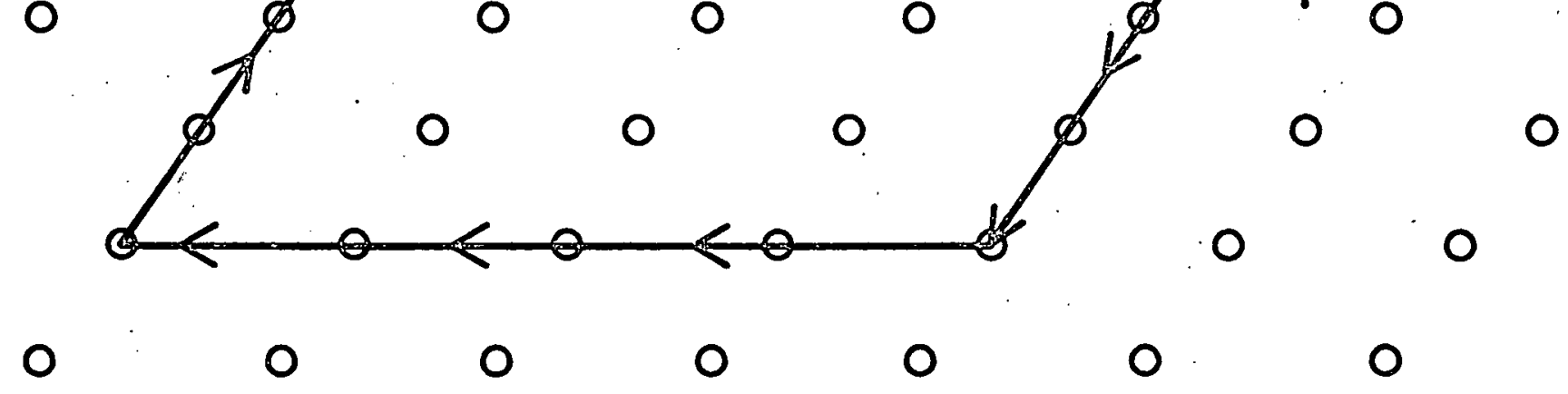

0

$\begin{array}{ccccccccc}0 & 0 & 0 & 0 & 0 & 0 & 0 \\ 0 & 0 & 0 & 0 & 0 & 0 & 0\end{array}$




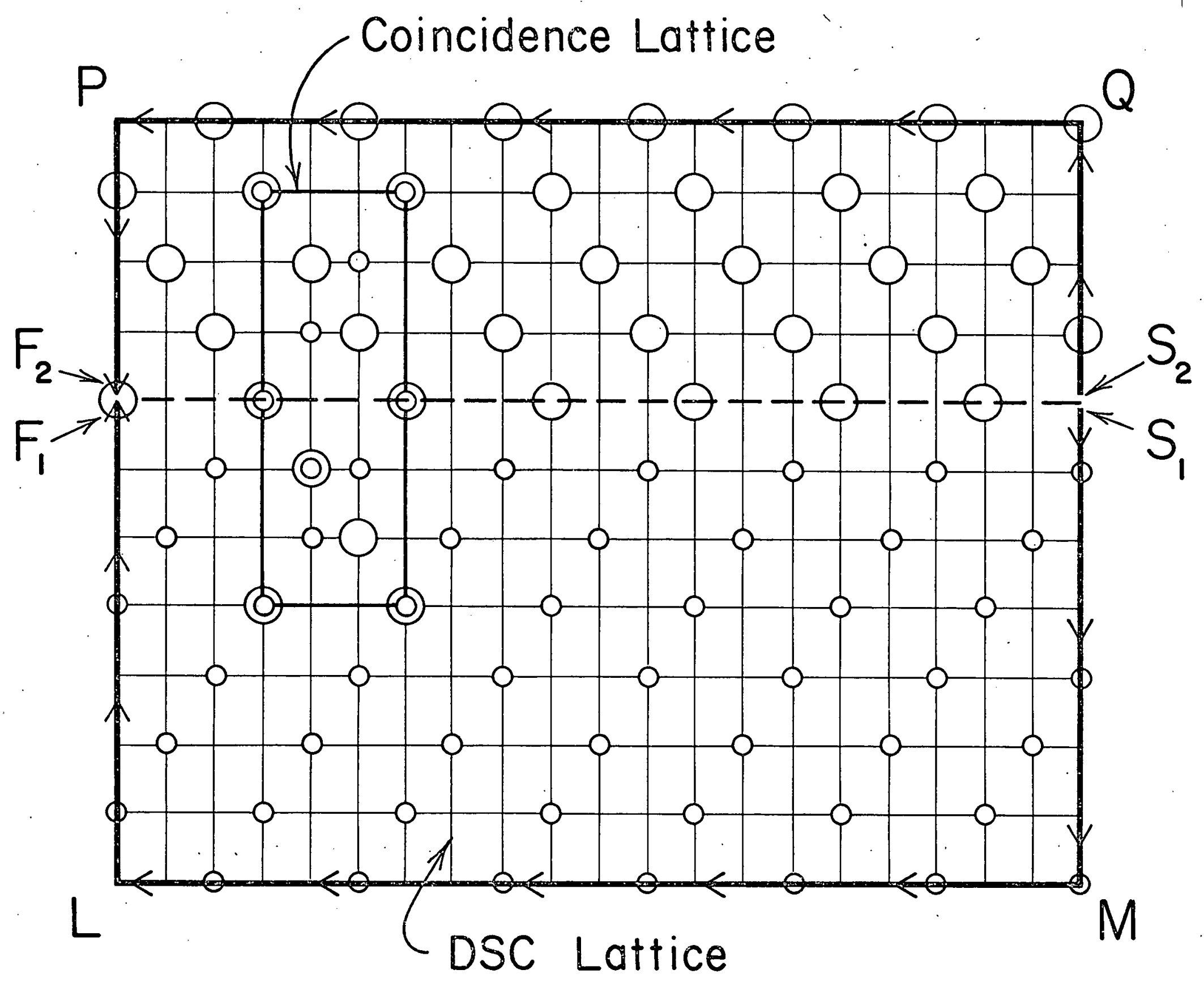




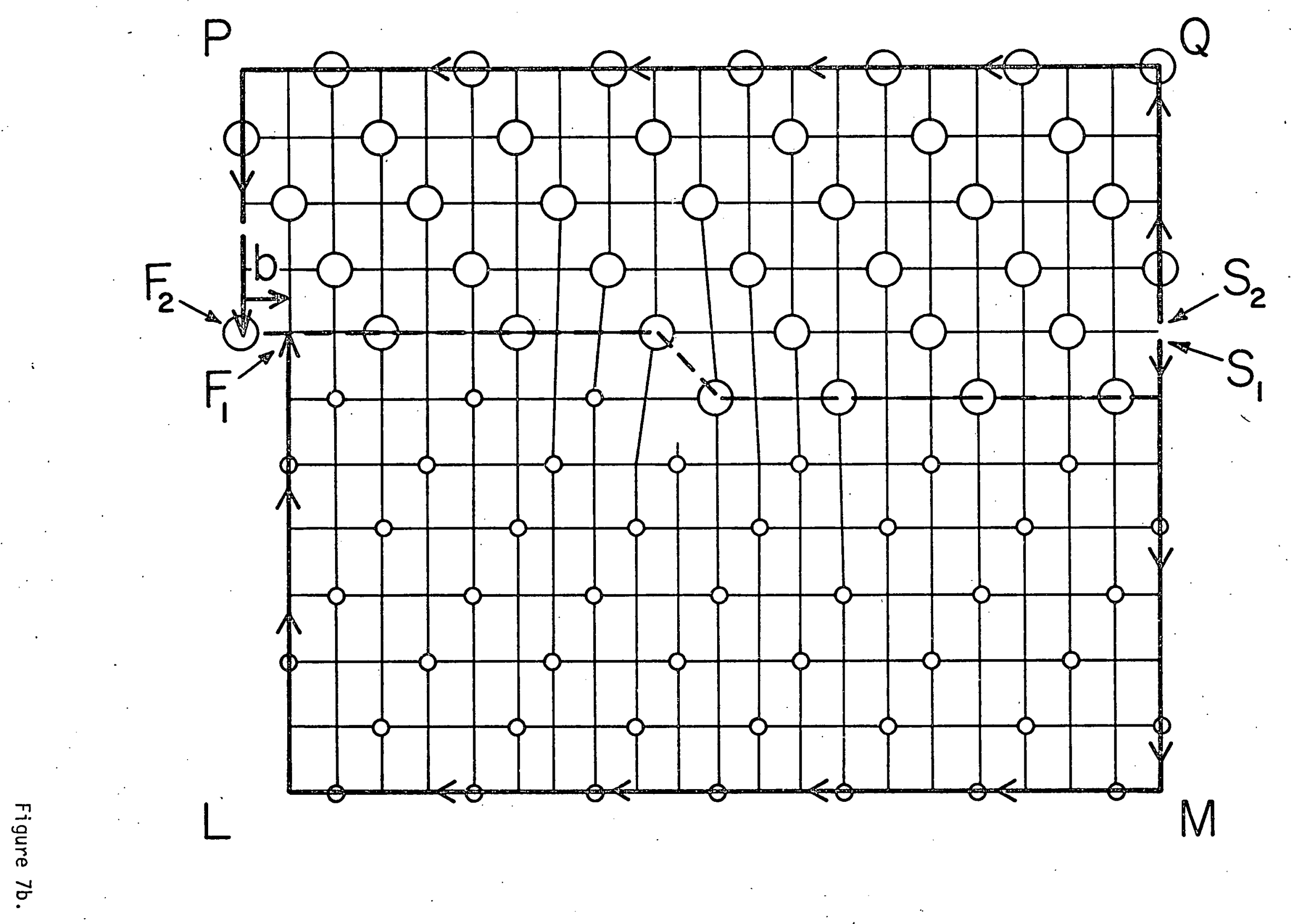




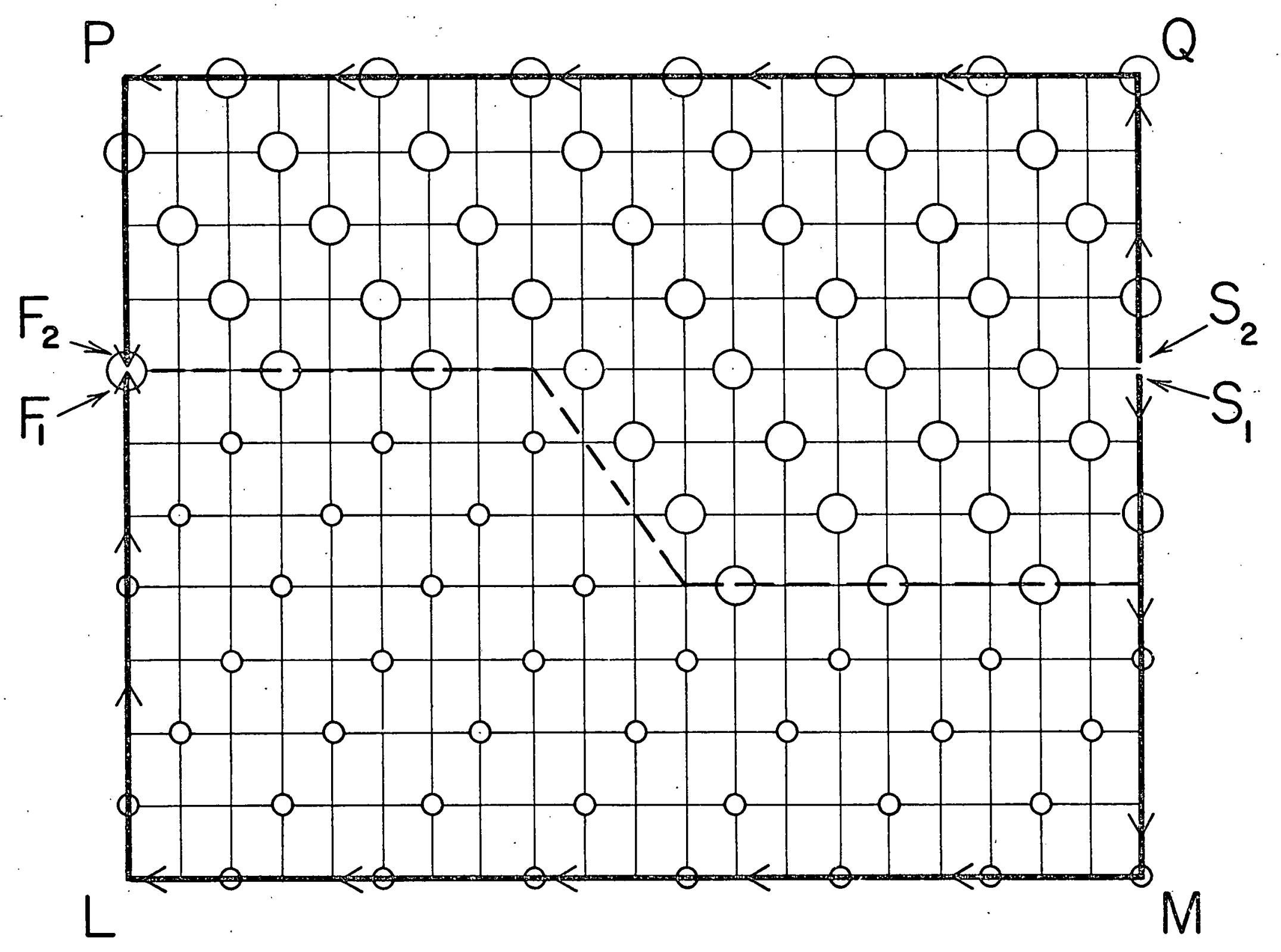

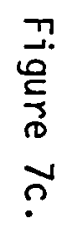




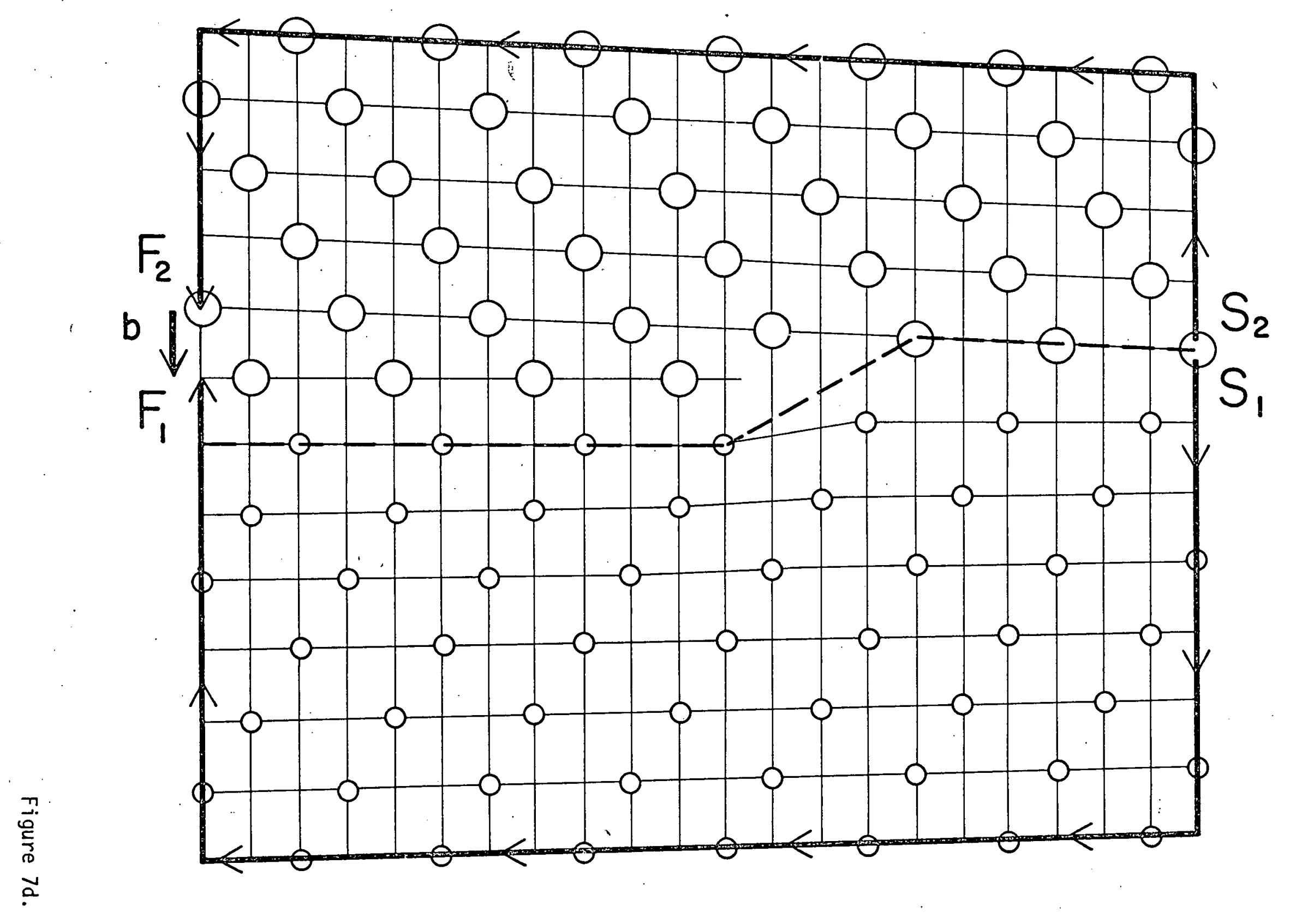




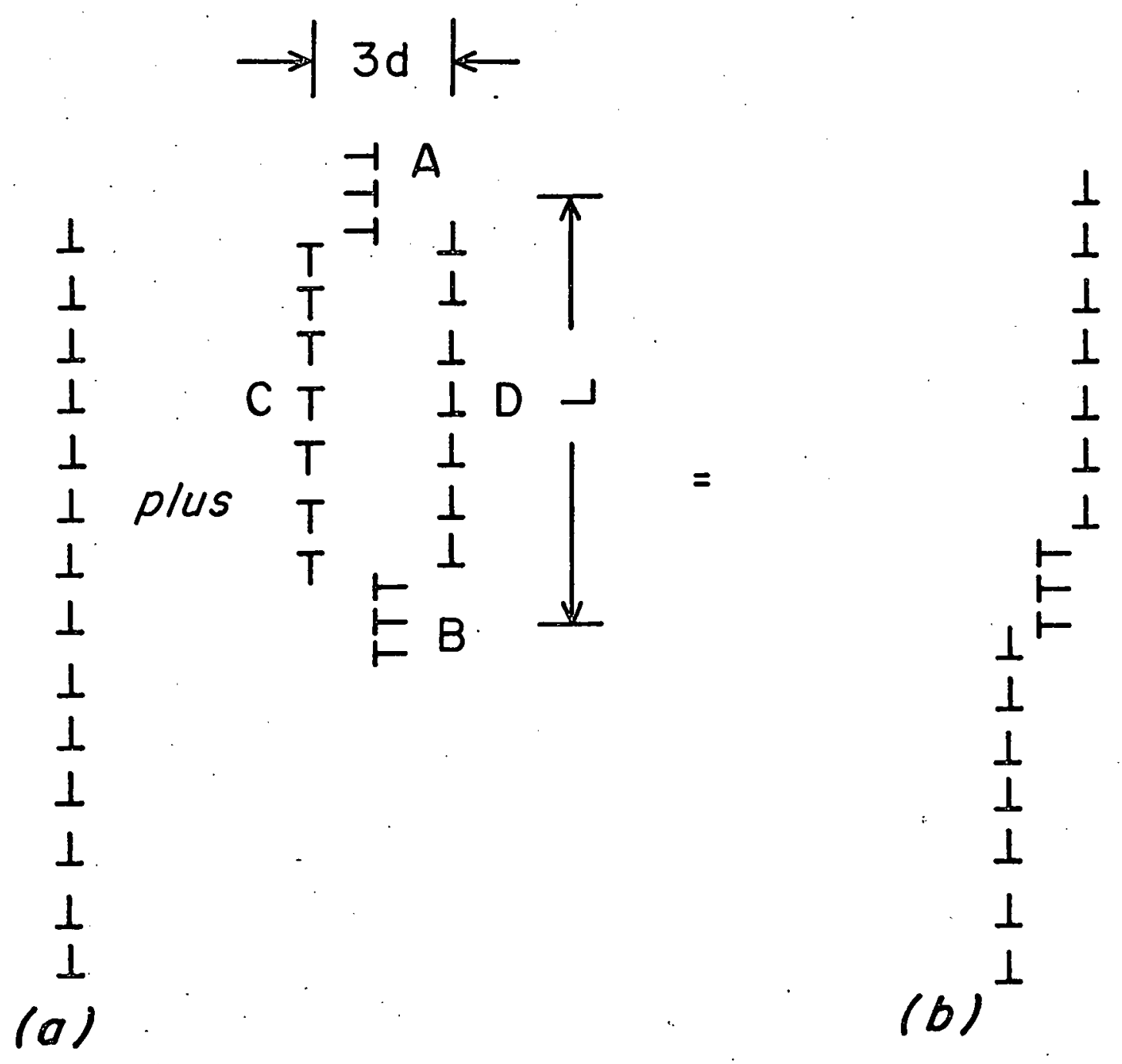

$$
\begin{aligned}
& \rightarrow \perp \\
& \rightarrow \perp \\
& \rightarrow \perp \\
& \longrightarrow \perp \\
& \perp \\
& 1 \\
& \frac{1}{1} \\
& \frac{1}{1} \mathrm{~F} \\
& \frac{1}{1} \\
& (c)
\end{aligned}
$$

Figure 8. 


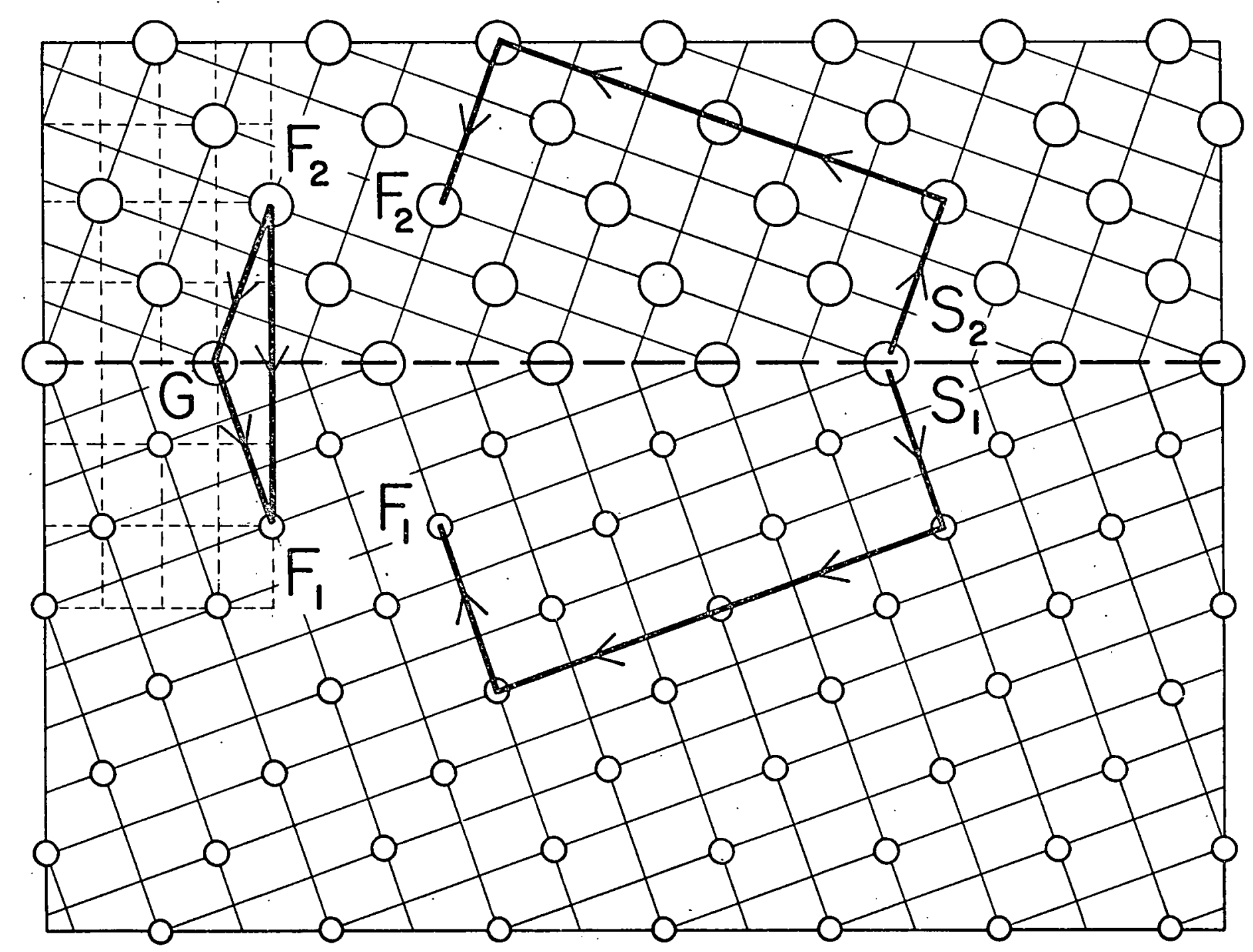




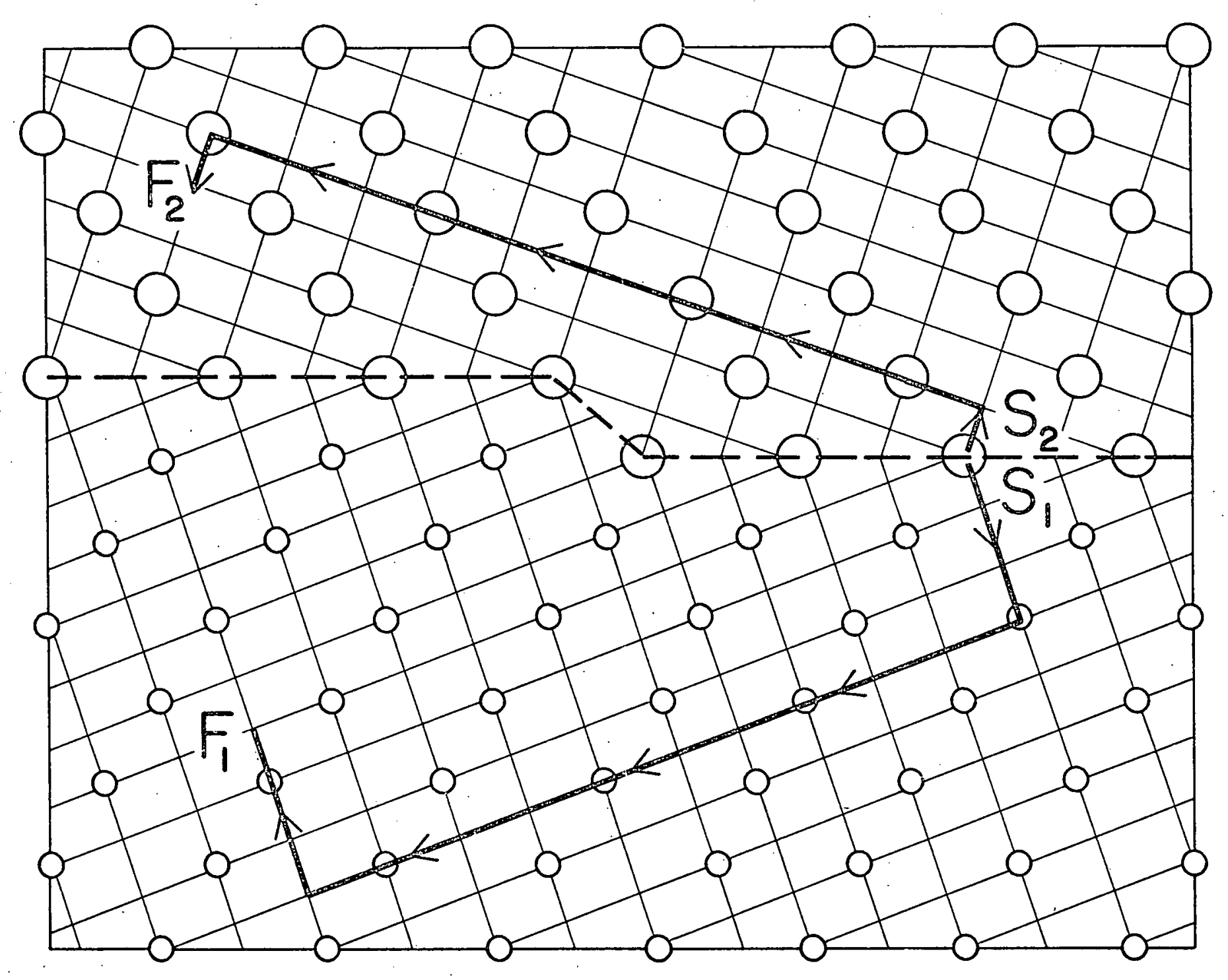




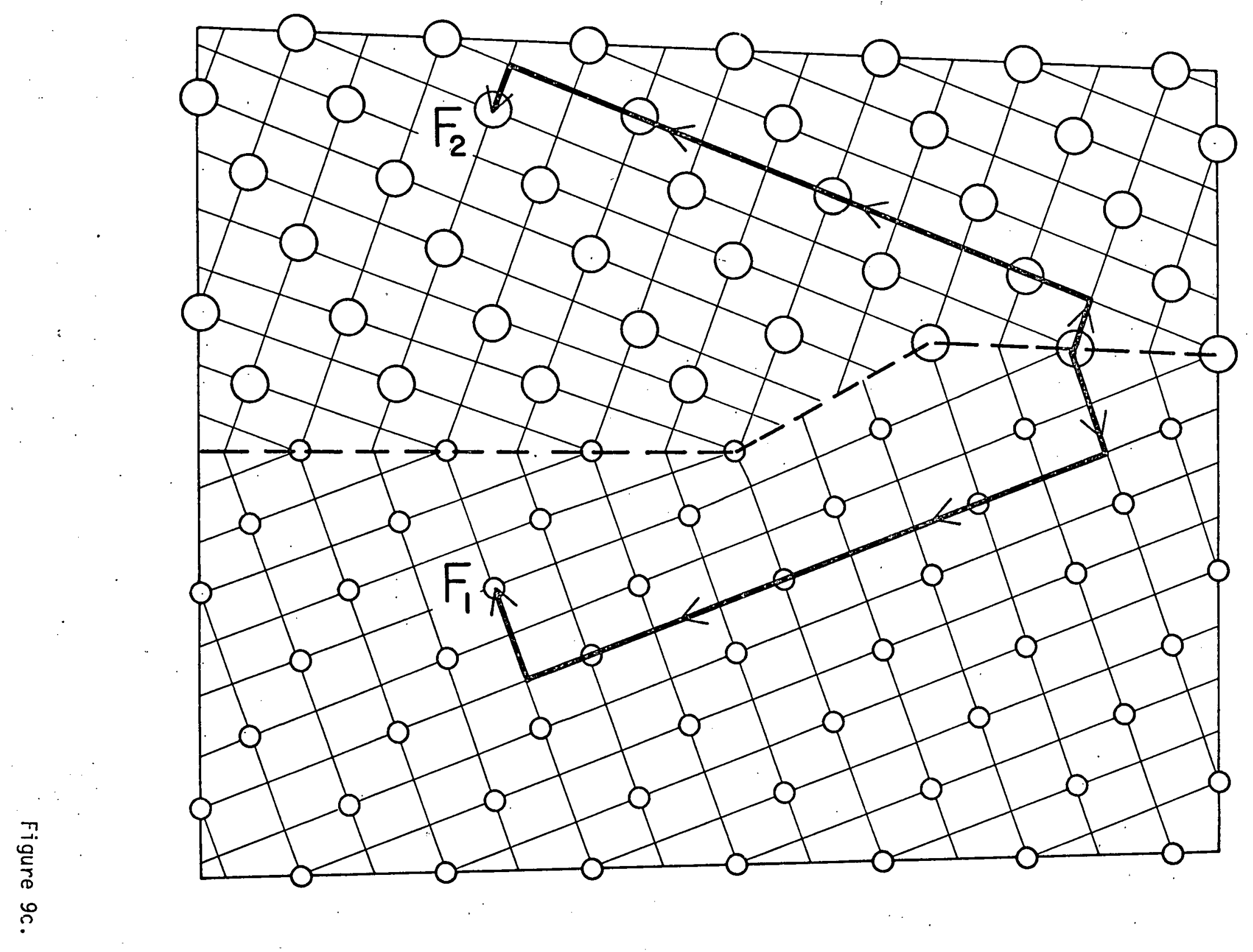




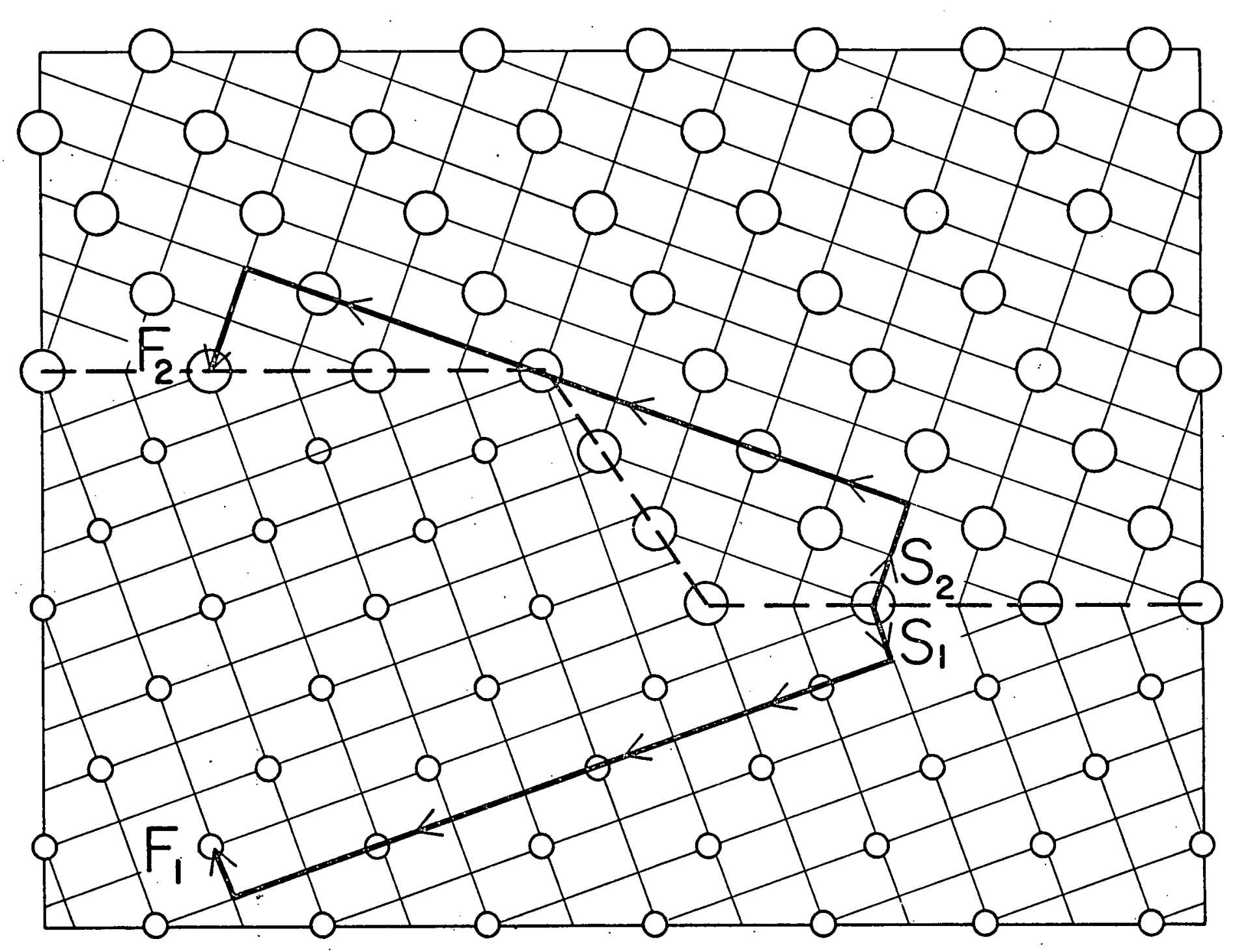

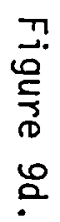




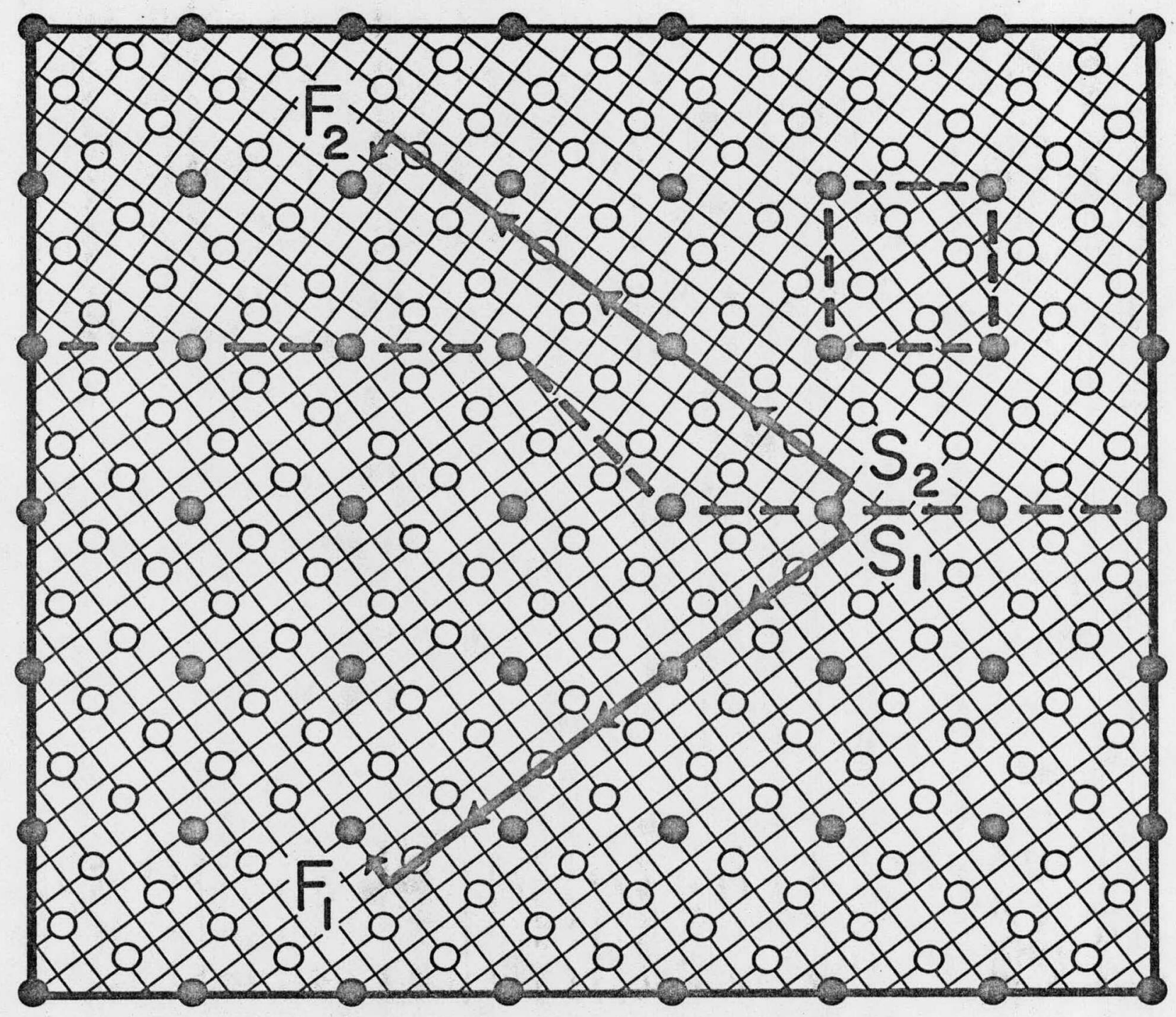


1

$\perp$

1

$\perp$

$\perp$

$\perp$

$\perp$
1

$\perp$

$\perp$

$\perp$

$\therefore$

$\perp$

$\perp$ 


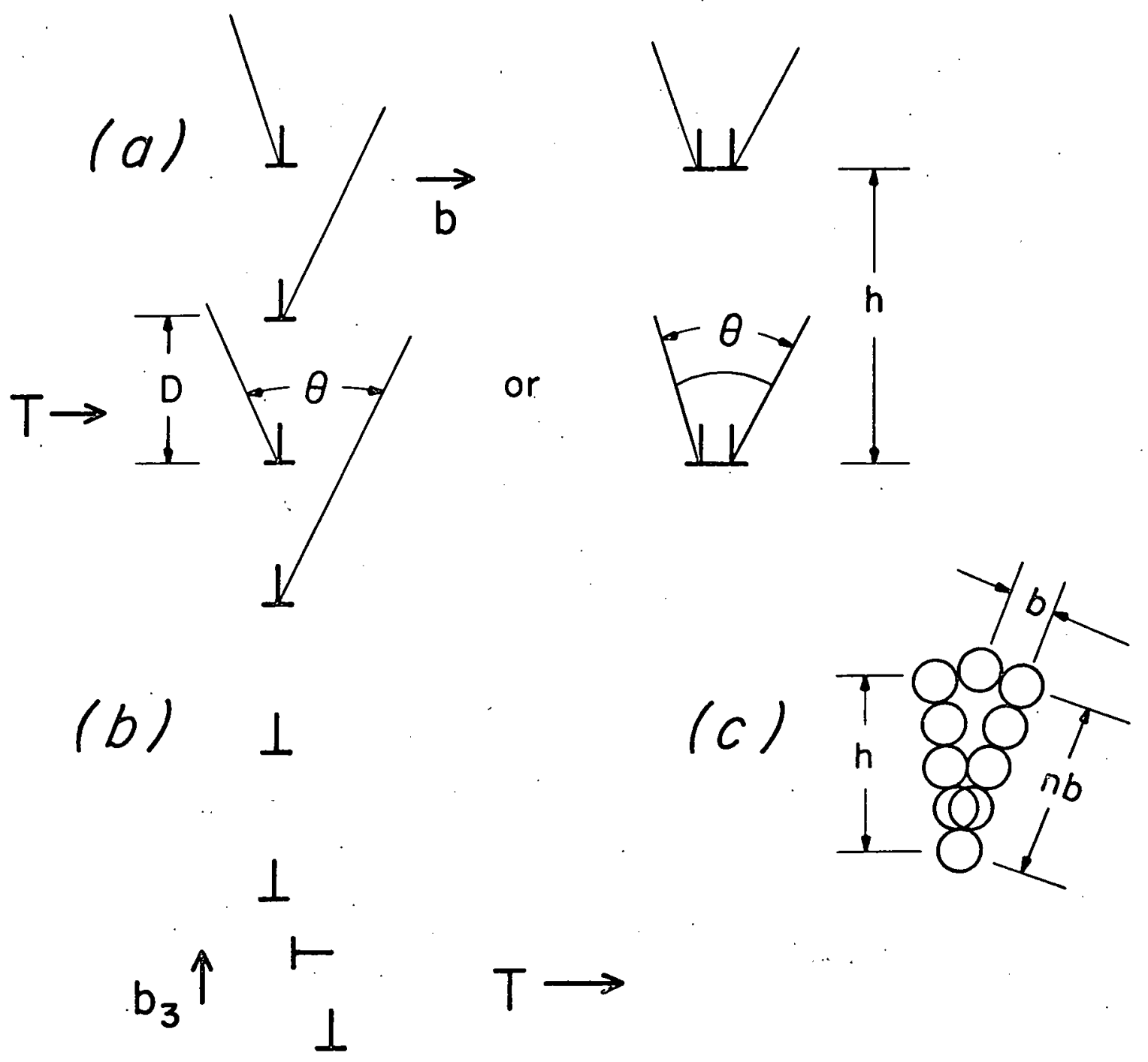

$\perp$

Figure 12. 


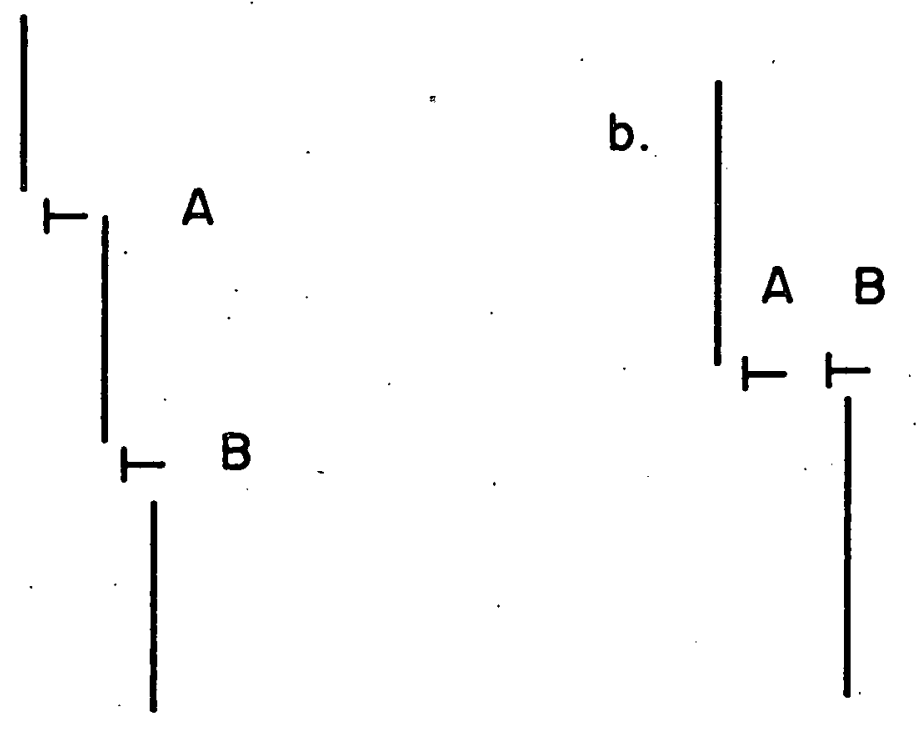

Figure 13. 